\title{
One-Pot Enol Silane Formation-Mukaiyama Aldol-Type Addition to Dimethyl Acetals Mediated by TMSOTf
}

\author{
C. Wade Downey,* Miles W. Johnson, and Kathryn J. Tracy \\ Department of Chemistry, University of Richmond, Richmond, Virginia 23173
}

\section{Supporting Information}

\section{Contents}

\section{General Information \\ Experimental Procedures \\ NMR Spectra \\ 1 \\ 1-7 \\ 8-32}

See main text for typical procedure.

General. Reactions were carried out under an atmosphere of nitrogen with a septum cap in oven-dried glassware with magnetic stirring. $\mathrm{CH}_{2} \mathrm{Cl}_{2}$ was purified by passage through a bed of activated alumina, ${ }^{1}$ or used as received from Aldrich Chemical Company. Aryl ketones and esters were distilled and stored under inert atmosphere, except for 2-acetonaphthone, which was used as received. TMSOTf was stored in a Schlenk flask under inert atmosphere. TESOTf was used as received. Thioesters and amides were synthesized in accord with literature precedent. ${ }^{2}$ Dimethoxymethane, propionaldehyde dimethyl acetal, benzaldehyde dimethyl acetal, p-anisaldehyde dimethyl acetal, acetaldehyde dimethyl acetal, acetaldehyde diethyl acetal, and 2-methoxypyran were used as received. All other acetals were synthesized in accord with literature precedent. ${ }^{3}$ Amine bases were distilled and stored in a Schlenk flask under inert atmosphere. Purification of reaction products was carried out by flash chromatography using silica gel (230-400 mesh). Analytical thin layer chromatography was performed on silica gel plates. Visualization was accomplished with UV light and anisaldehyde stain, followed by heating. Infrared spectra were recorded on an FT-IR spectrometer. ${ }^{1} \mathrm{H}$ NMR spectra were recorded on a 500 $\mathrm{MHz}$ spectrometer or $300 \mathrm{MHz}$ spectrometer, and are reported in ppm using solvent as an internal standard $\left(\mathrm{CDCl}_{3}\right.$ at $\left.7.28 \mathrm{ppm}\right)$. Data are reported as $(\mathrm{ap}=$ apparent, $\mathrm{s}=$ singlet, $\mathrm{d}=$ doublet, $\mathrm{t}=$ triplet, $\mathrm{q}=\mathrm{quartet}, \mathrm{sx}=$ sextet, $\mathrm{sp}=$ septet, $\mathrm{m}=$ multiplet, $\mathrm{b}=$ broad; coupling constant(s) in $\mathrm{Hz}$; integration). Proton-decoupled ${ }^{13} \mathrm{C} \mathrm{NMR}$ spectra were recorded on a $125 \mathrm{MHz}$ spectrometer or $75 \mathrm{MHz}$ spectrometer, and are reported in ppm using solvent as an internal standard $\left(\mathrm{CDCl}_{3}\right.$ at $\left.77.0 \mathrm{ppm}\right)$. High-resolution mass spectra were obtained by electrospray ionization. Melting points were determined using a capillary melting point apparatus.

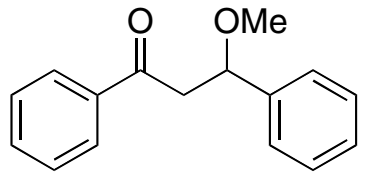

3-Methoxy-1,3-diphenylpropan-1-one (1) The title compound ${ }^{4}$ was prepared according to the General Procedure, using acetophenone $(117 \mu \mathrm{L}, 120 \mathrm{mg}, 1.00 \mathrm{mmol})$ as the enolate precursor and benzaldehyde dimethyl acetal $(210 \mu \mathrm{L}, 213 \mathrm{mg}, 1.40 \mathrm{mmol})$ as the acetal. The product was isolated as a colorless oil (99\% yield): IR (film) 3055 , $3023,2925,2822,1681,1595,1459,1356,1269,1198,1095,992,748,699 \mathrm{~cm}^{-1} ;{ }^{1} \mathrm{H}$ NMR $\left(500 \mathrm{MHz}, \mathrm{CDCl}_{3}\right)$ $\delta$ 8.00-7.95 $(\mathrm{m}, 2 \mathrm{H}), 7.59-7.55(\mathrm{~m}, 1 \mathrm{H}), 7.49-7.37(\mathrm{~m}, 6 \mathrm{H}), 7.35-7.30(\mathrm{~m}, 1 \mathrm{H}), 4.91(\mathrm{dd}, J=4.5,8.6 \mathrm{~Hz}, 1 \mathrm{H})$, $3.62(\mathrm{dd}, J=8.5,16.8 \mathrm{~Hz}, 1 \mathrm{H}), 3.26(\mathrm{~s}, 3 \mathrm{H}), 3.41(\mathrm{dd}, J=4.4,16.2 \mathrm{~Hz}, 1 \mathrm{H}) ;{ }^{13} \mathrm{C} \mathrm{NMR}\left(125 \mathrm{MHz} \mathrm{CDCl}_{3}\right)$

(1) Pangborn, A. B.; Giardello, M. A.; Grubbs, R. H.; Rosen, R. K.; Timmers, F. J. Organometallics 1996, 15, 1518-1520.

(2) Ranu, B. C.; Dey, S. S.; Hajra, A. Green Chem. 2003, 5, 44-46.

(3) Clerici, A.; Pastori, N.; Obretta, P. Tetrahedron 1998, 54, 15679-15690.

(4) For a literature synthesis of product 1, including NMR data, see: Torii, S.; Inokuchi, T.; Takagishi, S.; Horike, H.; Kuroda, H.; Uneyama, K. Bull. Chem. Soc. Jpn. 1987, 60, 2173-2188. 
$\delta 197.7,141.5,137.3,133.1,128.6,128.5,128.2,127.9,126.1,79.6,56.9,47.2 ;$ HRMS (ESI): Exact mass calcd for $\mathrm{C}_{16} \mathrm{H}_{16} \mathrm{O}_{2} \mathrm{Na}[\mathrm{M}+\mathrm{Na}]^{+}, 263.1043$. Found 263.1040 .

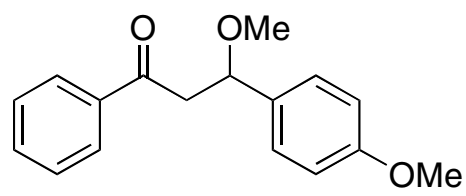

3-Methoxy-(3-(4-methoxyphenyl)-1-phenylpropan-1-one (2) The title compound was prepared according to the General Procedure, using acetophenone $(117 \mu \mathrm{L}, 120 \mathrm{mg}, 1.00 \mathrm{mmol})$ as the enolate precursor and anisaldehyde dimethyl acetal $(235 \mu \mathrm{L}, 251 \mathrm{mg}, 1.40 \mathrm{mmol})$ as the acetal. The product was isolated as a pale yellow oil (99\% yield): IR (film) 2925, 2241, 1681, 1600, 1502, 1242, 1160, 1095, 905, $720 \mathrm{~cm}^{-1} ;{ }^{1} \mathrm{H}$ NMR (300 MHz, $\left.\mathrm{CDCl}_{3}\right) \delta$ 7.99-7.93 (m, 2H), 7.60-7.53 (m, 1H), 7.50-7.42 (m, 2H), 7.38-7.31 (m, 2H), 6.96-6.89 (m, 2H), $4.84(\mathrm{dd}, J=4.8,8.2 \mathrm{~Hz}, 1 \mathrm{H}), 3.83(\mathrm{~s}, 3 \mathrm{H}), 3.61(\mathrm{dd}, J=8.1,16.5 \mathrm{~Hz}$, $1 \mathrm{H}), 3.23(\mathrm{~s}, 3 \mathrm{H}), 3.10(\mathrm{dd}, J=4.7,16.6 \mathrm{~Hz}, 1 \mathrm{H}) ;{ }^{13} \mathrm{C}$ NMR $\left(125 \mathrm{MHz}, \mathrm{CDCl}_{3}\right) \delta 197.8,159.3,137.3,133.4$, 133.0, 128.5, 128.2, 127.9, 114.0, 79.2, 56.6, 55.3, 47.1; HRMS (ESI): Exact mass calcd for $\mathrm{C}_{17} \mathrm{H}_{18} \mathrm{O}_{3} \mathrm{Na}$ $[\mathrm{M}+\mathrm{Na}]^{+}, 293.1148$. Found 293.1153.

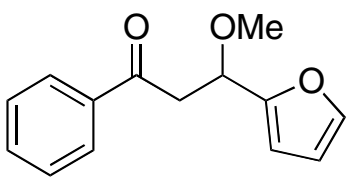

3-(2-Furyl)-3-methoxy-1-phenylpropan-1-one (3) The title compound was prepared according to the General Procedure, using acetophenone (117 $\mu \mathrm{L}, 120 \mathrm{mg}, 1.00 \mathrm{mmol})$ as the enolate precursor and 2-furanaldehyde dimethyl acetal $(189 \mu \mathrm{L}, 199 \mathrm{mg}, 1.40 \mathrm{mmol})$ as the acetal. The product was isolated as an orange oil (88\% yield): IR (film) 2932 , $2819,1678,1592,1499,1441,1359,1282,1204,1095,1006,917,746,683 \mathrm{~cm}^{-1} ;{ }^{1} \mathrm{H} \mathrm{NMR}\left(500 \mathrm{MHz}, \mathrm{CDCl}_{3}\right)$ $\delta$ 8.02-7.98 (m, 2H), 7.61-7.56 (m, 1H), 7.51-7.46 (m, 2H), 7.44-7.42 (m, 1H), 6.40-6.35 (m, 2H), $4.95(\mathrm{dd}, J=$ $5.0,7.9 \mathrm{~Hz}, 1 \mathrm{H}), 3.73(\mathrm{dd}, J=7.8,16.5 \mathrm{~Hz}, 1 \mathrm{H}), 3.33(\mathrm{dd}, J=5.0,16.4 \mathrm{~Hz}, 1 \mathrm{H}), 3.32(\mathrm{~s}, 3 \mathrm{H}) ;{ }^{13} \mathrm{C} \mathrm{NMR}(125$ $\left.\mathrm{MHz}, \mathrm{CDCl}_{3}\right) \delta 197.1,153.3,142.6,137.1,133.2,128.6,128.2,110.1,108.4,72.4,56.8,43.0$; HRMS (ESI): Exact mass calcd for $\mathrm{C}_{14} \mathrm{H}_{14} \mathrm{O}_{3} \mathrm{Na}[\mathrm{M}+\mathrm{Na}]^{+}, 253.0835$. Found 253.0824.

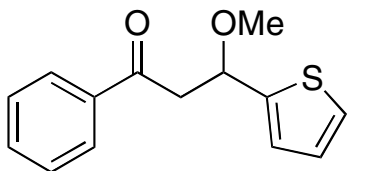

3-Methoxy-1-phenyl-3-(2-thienyl)propan-1-one (4) The title compound was prepared according to the General Procedure, using acetophenone $(117 \mu \mathrm{L}, 120 \mathrm{mg}, 1.00 \mathrm{mmol})$ as the enolate precursor and 2-thiophene carboxaldehyde dimethyl acetal $(198 \mu \mathrm{L}, 221 \mathrm{mg}$, $1.40 \mathrm{mmol})$ as the acetal. The product was isolated as an orange oil (95\% yield): IR (film) 2936, 1681, 1595, 1443, 1361, 1280, 1220, 1100, 981, 824, 758, $699 \mathrm{~cm}^{-1}$; ${ }^{1} \mathrm{H}$ NMR $\left(300 \mathrm{MHz}, \mathrm{CDCl}_{3}\right)$ $\delta 8.00-7.95(\mathrm{~m}, 2 \mathrm{H}), 7.62-7.54(\mathrm{~m}, 1 \mathrm{H}), 7.51-7.44(\mathrm{~m}, 2 \mathrm{H}), 7.32-7.29(\mathrm{~m}, 1 \mathrm{H}), 7.10-7.06(\mathrm{~m}, 1 \mathrm{H}), 7.00(\mathrm{dd}, J=$ $3.6,5.1 \mathrm{~Hz}, 1 \mathrm{H}), 5.19(\mathrm{dd}, J=5.0,8.2 \mathrm{~Hz}, 1 \mathrm{H}), 3.70(\mathrm{dd}, J=8.0,16.5 \mathrm{~Hz}, 1 \mathrm{H}), 3.32(\mathrm{~s}, 3 \mathrm{H}), 3.26(\mathrm{dd}, J=4.7$, $16.6 \mathrm{~Hz}, 1 \mathrm{H}) ;{ }^{13} \mathrm{C}$ NMR $\left(75 \mathrm{MHz}, \mathrm{CDCl}_{3}\right) \delta 197.2,145.2,137.1,133.2,128.6,128.2,126.6,125.5,125.1,75.2$, 56.9, 47.2; HRMS (ESI): Exact mass calcd for $\mathrm{C}_{14} \mathrm{H}_{14} \mathrm{O}_{\mathrm{s}} \mathrm{SNa}[\mathrm{M}+\mathrm{Na}]^{+}, 269.0607$. Found 269.0604.

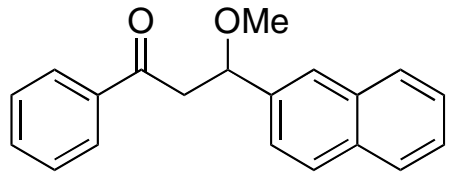

3-Methoxy-3-(2-naphthyl)-1-phenylpropan-1-one (5) The title compound was prepared according to the General Procedure, using acetophenone $(117 \mu \mathrm{L}, 120$ $\mathrm{mg}, 1.00 \mathrm{mmol}$ ) as the enolate precursor and 2-naphthaldehyde dimethyl acetal $(230 \mu \mathrm{L}, 250 \mathrm{mg}, 1.40 \mathrm{mmol})$ as the acetal. The product was isolated as an amorphous white solid (99\% yield): mp: $96-99{ }^{\circ} \mathrm{C}$; IR (film) 3055, 2925, 2822, 1681, 1595, 1437, 1356, 1269, 1198, 1095, 981, 905, $813 \mathrm{~cm}^{-1} ;{ }^{1} \mathrm{H}$ NMR $\left(500 \mathrm{MHz}, \mathrm{CDCl}_{3}\right) \delta 8.02-7.98(\mathrm{~m}, 2 \mathrm{H}), 7.93-7.86(\mathrm{~m}, 4 \mathrm{H}), 7.61-7.55$ $(\mathrm{m}, 2 \mathrm{H}), 7.55-7.49(\mathrm{~m}, 2 \mathrm{H}), 7.49-7.45(\mathrm{~m}, 2 \mathrm{H}), 5.11(\mathrm{dd}, J=4.5,8.7 \mathrm{~Hz}, 1 \mathrm{H}), 3.73(\mathrm{dd}, J=8.6,16.6 \mathrm{~Hz}, 1 \mathrm{H})$, $3.33(\mathrm{~s}, 3 \mathrm{H}), 3.21(\mathrm{dd}, J=4.4,16.8 \mathrm{~Hz}, 1 \mathrm{H}) ;{ }^{13} \mathrm{C} \mathrm{NMR}\left(125 \mathrm{MHz}, \mathrm{CDCl}_{3}\right) \delta 197.6,138.9,137.3,133.3,133.2$, 133.1, 128.6, 128.6, 128.3, 128.0, 127.8, 126.3, 126.0, 125.9, 124.4, 79.8, 57.0, 47.1; HRMS (ESI): Exact mass calcd for $\mathrm{C}_{20} \mathrm{H}_{18} \mathrm{O}_{2} \mathrm{Na}[\mathrm{M}+\mathrm{Na}]^{+}, 313.1199$. Found 313.1205. 


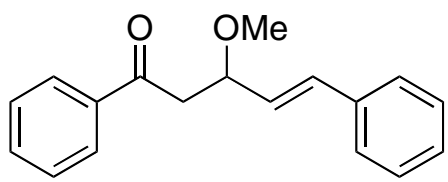

(4E)-3-Methoxy-1,5-diphenylpent-4-en-1-one (6) The title compound was prepared according to the General Procedure, except that the reaction was run for 3 h. Acetophenone $(117 \mu \mathrm{L}, 120 \mathrm{mg}, 1.00 \mathrm{mmol})$ was used as the enolate precursor and cinnamaldehyde dimethyl acetal $(248 \mathrm{mg}, 1.40 \mathrm{mmol})$ as the acetal. The product was isolated as a yellow oil (99\% yield): IR (film) 2928, 2350, 1685, 1445, 1351, 1208, 1087, 967, 750, $687 \mathrm{~cm}^{-1} ;{ }^{1} \mathrm{H}$ NMR $\left(500 \mathrm{MHz}, \mathrm{CDCl}_{3}\right) \delta 8.01-7.98(\mathrm{~m}, 2 \mathrm{H}), 7.61-7.56(\mathrm{~m}, 1 \mathrm{H}), 7.51-7.46(\mathrm{~m}, 2 \mathrm{H}), 7.44-7.40(\mathrm{~m}$, $2 \mathrm{H}), 7.37-7.32(\mathrm{~m}, 2 \mathrm{H}), 7.30-7.25(\mathrm{~m}, 1 \mathrm{H}), 6.69(\mathrm{~d}, J=15.9,1 \mathrm{H}), 6.19(\mathrm{dd}, J=7.3,15.9 \mathrm{~Hz}, 1 \mathrm{H}), 4.52-4.46(\mathrm{~m}$, $1 \mathrm{H}), 3.49(\mathrm{dd}, J=7.4,16.3 \mathrm{~Hz}, 1 \mathrm{H}), 3.37(\mathrm{~s}, 3 \mathrm{H}), 3.10(\mathrm{dd}, J=5.2,16.4 \mathrm{~Hz}, 1 \mathrm{H}) ;{ }^{13} \mathrm{C} \mathrm{NMR}\left(125 \mathrm{MHz}, \mathrm{CDCl}_{3}\right)$ $\delta$ 197.7, 137.3, 136.4, 133.1, 132.6, 128.9, 128.6, 128.6, 128.3, 127.9, 126.6, 78.4, 56.7, 44.9; HRMS (ESI): Exact mass calcd for $\mathrm{C}_{18} \mathrm{H}_{18} \mathrm{O}_{2} \mathrm{Na}[\mathrm{M}+\mathrm{Na}]^{+}, 289.1199$. Found 289.1209.

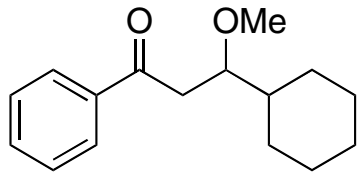

3-Cyclohexyl-3-methoxy-1-phenylpropan-1-one (7) The title compound was prepared according to the General Procedure, using acetophenone $(117 \mu \mathrm{L}, 120 \mathrm{mg}, 1.00 \mathrm{mmol})$ as the enolate precursor and cyclohexane carboxaldehyde dimethyl acetal $(240 \mu \mathrm{L}, 222$ $\mathrm{mg}, 1.40 \mathrm{mmol}$ ) as the acetal. The product was isolated as a pale yellow oil (91\% yield): IR (film) 2924, 2850, 1685, 1619, 1441, 1363, 1328, 1274, 1208, 1095, 1006, 975, 746, $691 \mathrm{~cm}^{-1}$; ${ }^{1} \mathrm{H} \mathrm{NMR}$ $\left(300 \mathrm{MHz} \mathrm{CDCl}_{3}\right) \delta 8.02-7.96(\mathrm{~m}, 2 \mathrm{H}), 7.62-7.54(\mathrm{~m}, 1 \mathrm{H}), 7.52-7.44(\mathrm{~m}, 2 \mathrm{H}), 3.74(\mathrm{ap} \mathrm{dt}, J=4.6,9.2 \mathrm{~Hz}, 1 \mathrm{H})$, $3.34(\mathrm{~s}, 3 \mathrm{H}), 3.24(\mathrm{dd}, J=7.8,16.2 \mathrm{~Hz}, 1 \mathrm{H}), 2.95(\mathrm{dd}, J=4.0,16.3 \mathrm{~Hz}, 1 \mathrm{H}), 1.90-1.57(\mathrm{~m}, 6 \mathrm{H}), 1.36-1.00(\mathrm{~m}$, $5 \mathrm{H}) ;{ }^{13} \mathrm{C}$ NMR $\left(75 \mathrm{MHz}, \mathrm{CDCl}_{3}\right) \delta 199.6,137.5,133.0,128.6,128.2,81.9,58.3,42.0,40.8,28.7,28.5,26.6$, 26.3; HRMS (ESI): Exact mass calcd for $\mathrm{C}_{16} \mathrm{H}_{22} \mathrm{O}_{2} \mathrm{Na}[\mathrm{M}+\mathrm{Na}]^{+}, 269.1512$. Found 269.1503.

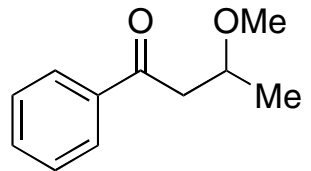

3-Methoxy-1-phenylbutan-1-one (8) The title compound was prepared according to the General Procedure, using acetophenone $(117 \mu \mathrm{L}, 120 \mathrm{mg}, 1.00 \mathrm{mmol})$ as the enolate precursor and acetaldehyde dimethyl acetal $(127 \mu \mathrm{L}, 108 \mathrm{mg}, 1.20 \mathrm{mmol})$ as the acetal. The product was isolated as a colorless oil (89\% yield): IR (film) 2968, 2925, 2816, 1676, 1595, 1437, 1367, 1296, 1204, 1079, $682 \mathrm{~cm}^{-1} ;{ }^{1} \mathrm{H}$ NMR (500 MHz, $\left.\mathrm{CDCl}_{3}\right) \delta 8.01-7.97(\mathrm{~m}, 2 \mathrm{H}), 7.62-7.56(\mathrm{~m}, 1 \mathrm{H})$, $7.52-7.46(\mathrm{~m}, 2 \mathrm{H}), 3.74(\mathrm{ap} \mathrm{dq}, J=6.0,6.4 \mathrm{~Hz}, 1 \mathrm{H}), 3.37(\mathrm{~s}, 3 \mathrm{H}), 3.36(\mathrm{dd}, J=6.6,15.9 \mathrm{~Hz}, 1 \mathrm{H}), 2.94(\mathrm{dd}, J=$ $6.5,16.4 \mathrm{~Hz}, 1 \mathrm{H}), 1.28(\mathrm{~d}, J=6.5 \mathrm{~Hz}, 3 \mathrm{H}) ;{ }^{13} \mathrm{C} \mathrm{NMR}\left(125 \mathrm{MHz}, \mathrm{CDCl}_{3}\right) \delta 198.7,137.4,133.1,128.6,128.2$, 73.6, 56.4, 45.5, 19.6; HRMS (ESI): Exact mass calcd for $\mathrm{C}_{11} \mathrm{H}_{14} \mathrm{O}_{2} \mathrm{Na}[\mathrm{M}+\mathrm{Na}]^{+}$, 201.0886. Found 201.0892.

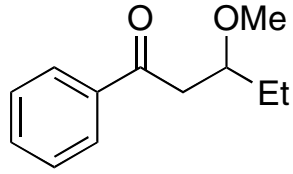

3-Methoxy-1-phenylpentan-1-one (9) The title compound was prepared according to the General Procedure, using acetophenone $(117 \mu \mathrm{L}, 120 \mathrm{mg}, 1.00 \mathrm{mmol})$ as the enolate precursor and propionaldehyde dimethyl acetal $(174 \mu \mathrm{L}, 146 \mathrm{mg}, 1.40 \mathrm{mmol})$ as the acetal. The product was isolated as a colorless oil (90\% yield): IR (film) 2963, 2924, 2870, 1678, 1592, 1441, 1359, 1208, 1080, 1002, 746, $683 \mathrm{~cm}^{-1} ;{ }^{1} \mathrm{H}$ NMR $\left(500 \mathrm{MHz}, \mathrm{CDCl}_{3}\right) \delta 8.01-7.97(\mathrm{~m}, 2 \mathrm{H}), 7.61-$ $7.56(\mathrm{~m}, 1 \mathrm{H}), 7.52-7.46(\mathrm{~m}, 2 \mathrm{H}), 3.89-3.82(\mathrm{~m}, 1 \mathrm{H}), 3.37(\mathrm{~s}, 3 \mathrm{H}), 3.30(\mathrm{dd}, J=6.8,15.9 \mathrm{~Hz}, 1 \mathrm{H}), 2.96(\mathrm{dd}, J=$ $5.0,15.8 \mathrm{~Hz}, 1 \mathrm{H}), 1.70-1.60(\mathrm{~m}, 2 \mathrm{H}), 0.97(\mathrm{t}, J=7.6 \mathrm{~Hz}, 3 \mathrm{H}) ;{ }^{13} \mathrm{C}$ NMR $\left(125 \mathrm{MHz}, \mathrm{CDCl}_{3}\right) \delta 199.1,137.5$, 133.0, 128.6, 128.2, 78.7, 57.2, 42.9, 26.9, 9.4; HRMS (ESI): Exact mass calcd for $\mathrm{C}_{12} \mathrm{H}_{16} \mathrm{O}_{2} \mathrm{Na}[\mathrm{M}+\mathrm{Na}]^{+}$, 215.1043. Found 215.1035.

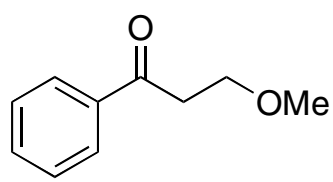

3-Methoxy-1-phenylpropan-1-one (10) The title compound ${ }^{5}$ was prepared according to the General Procedure, using acetophenone $(117 \mu \mathrm{L}, 120 \mathrm{mg}, 1.00 \mathrm{mmol})$ as the enolate precursor and dimethoxymethane $(97 \mu \mathrm{L}, 83 \mathrm{mg}, 1.10 \mathrm{mmol})$ as the acetal, except: a slight excess of TMSOTf was necessary for high conversion $(253 \mu \mathrm{L}, 311 \mathrm{mg}, 1.40 \mathrm{mmol})$. The product was isolated as a yellow oil (76\% yield): IR (film) 2963, 2924, 2870, 1678, 1592, 1441, 1359, 1208, 1080, 1002, 746, $683 \mathrm{~cm}^{-1}$; ${ }^{1} \mathrm{H}$ NMR $\left(500 \mathrm{MHz}, \mathrm{CDCl}_{3}\right) \delta 8.01-7.97(\mathrm{~m}, 2 \mathrm{H}), 7.61-7.56(\mathrm{~m}, 1 \mathrm{H}), 7.51-7.46(\mathrm{~m}$, $2 \mathrm{H}), 3.85(\mathrm{t}, J=6.7 \mathrm{~Hz}, 2 \mathrm{H}), 3.40(\mathrm{~s}, 3 \mathrm{H}), 3.27(\mathrm{t}, J=6.4 \mathrm{~Hz}, 2 \mathrm{H}) ;{ }^{13} \mathrm{C} \mathrm{NMR}\left(125 \mathrm{MHz}, \mathrm{CDCl}_{3}\right) \delta 198.3$,

(5) For a synthesis of product 10, including NMR data, see: Heasley, V. L.; Louie, T. J.; Luttrull, D. K.; Millar, M. D.; Moore, H. B.; Nogales, D. F.; Sauerbrey, A. M.; Shevel, A. B.; Shibuya, T. Y.; Stanley, M. S.; Shellhamer, D. F. J. Org. Chem. 1988, 53, $2199-2204$. 
137.0, 133.2, 128.6, 128.1, 67.9, 58.9, 38.7; HRMS (ESI): Exact mass calcd for $\mathrm{C}_{10} \mathrm{H}_{12} \mathrm{O}_{2} \mathrm{Na}[\mathrm{M}+\mathrm{Na}]^{+}$, 187.0730. Found 187.0732.

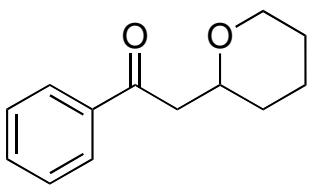

2-(2-pyranyl)-1-phenylethan-1-one (11) The title compound ${ }^{6}$ was prepared according to the General Procedure, using acetophenone $(117 \mu \mathrm{L}, 120 \mathrm{mg}, 1.00 \mathrm{mmol})$ as the enolate precursor and 2-methoxypyran $(133 \mu \mathrm{L}, 128 \mathrm{mg}, 1.10 \mathrm{mmol})$ as the acetal, except: a slight excess of TMSOTf was necessary for high conversion $(253 \mu \mathrm{L}, 311 \mathrm{mg}, 1.40 \mathrm{mmol})$. The product was isolated as a yellow oil (48\% yield):IR (film) 2930, 2846, 1674, 1595, 1447, 1276, 1206, 1095, 1049, 1002, 979, $747 \mathrm{~cm}^{-1}$; ${ }^{1} \mathrm{H}$ NMR (500 MHz, CDCl 3$) \delta 8.00-7.97$ (m, 2H), 7.60-7.55 (m, $1 \mathrm{H}), 7.50-7.45(\mathrm{~m}, 2 \mathrm{H}), 4.01-3.94(\mathrm{~m}, 2 \mathrm{H}), 3.53-3.46(\mathrm{~m}, 1 \mathrm{H}), 3.31(\mathrm{dd}, J=6.4,15.8,1 \mathrm{H}), 2.94(\mathrm{dd}, J=5.5$, $15.8 \mathrm{~Hz}, 1 \mathrm{H}), 1.92-1.82(\mathrm{~m}, 1 \mathrm{H}), 1.80-1.74(\mathrm{~m}, 1 \mathrm{H}), 1.67-1.48(\mathrm{~m}, 3 \mathrm{H}), 1.43-1.33(\mathrm{~m}, 1 \mathrm{H}) ;{ }^{13} \mathrm{C}$ NMR $(125$ $\left.\mathrm{MHz}, \mathrm{CDCl}_{3}\right) \delta 198.4,137.4,133.0,128.5,128.3,74.4,68.7,45.4,32.0,25.9,23.4 ;$ HRMS (ESI): Exact mass calcd for $\mathrm{C}_{13} \mathrm{H}_{16} \mathrm{O}_{2} \mathrm{Na}[\mathrm{M}+\mathrm{Na}]^{+}, 227.1043$. Found 227.1038.

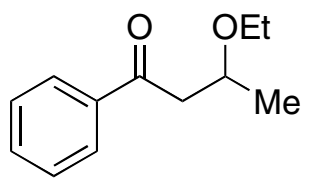

3-Ethoxy-1-phenylbutan-1-one (12) The title compound ${ }^{7}$ was prepared according to the General Procedure, using acetophenone $(117 \mu \mathrm{L}, 120 \mathrm{mg}, 1.00 \mathrm{mmol})$ as the enolate precursor and benzaldehyde diethyl acetal $(200 \mu \mathrm{L}, 165 \mathrm{mg}, 1.40 \mathrm{mmol})$ as the acetal, except: 2,6-lutidine $(139 \mu \mathrm{L}, 128 \mathrm{mg}, 1.20 \mathrm{mmol})$ was used instead of $i$ - $\operatorname{Pr}_{2} \mathrm{NEt}$. The product was isolated as a colorless oil (88\% yield): IR (film) 2975, 2920, 2866, 1678, 1592, 1445, 1363, 1293, 1204, 1087, 994, 746, $687 \mathrm{~cm}^{-1}$; ${ }^{1} \mathrm{H}$ NMR $\left(500 \mathrm{MHz}, \mathrm{CDCl}_{3}\right) \delta$ 8.00-7.97 (m, 2H), 7.60-7.55 (m, 1H), 7.50-7.46 $(\mathrm{m}, 2 \mathrm{H}), 4.11($ ap sx $J=6.1 \mathrm{~Hz}, 1 \mathrm{H}), 3.63-3.56(\mathrm{~m}, 1 \mathrm{H}), 3.51-3.43(\mathrm{~m}, 1 \mathrm{H}), 3.36(\mathrm{dd}, J=6.2,16.1 \mathrm{~Hz}, 1 \mathrm{H})$, $2.95(\mathrm{dd}, J=6.3,15.9 \mathrm{~Hz}, 1 \mathrm{H}), 1.28(\mathrm{~d}, J=6.0 \mathrm{~Hz}, 3 \mathrm{H}), 1.17(\mathrm{t}, J=7.4 \mathrm{~Hz}, 3 \mathrm{H}) ;{ }^{13} \mathrm{C} \mathrm{NMR}\left(125 \mathrm{MHz}, \mathrm{CDCl}_{3}\right)$ $\delta 198.9,137.4,133.0,128.5,128.2,71.9,64.2,45.9,20.4,15.5 ;$ HRMS (ESI): Exact mass calcd for $\mathrm{C}_{12} \mathrm{H}_{16} \mathrm{O}_{2} \mathrm{Na}[\mathrm{M}+\mathrm{Na}]^{+}, 215.1043$. Found 215.1038.

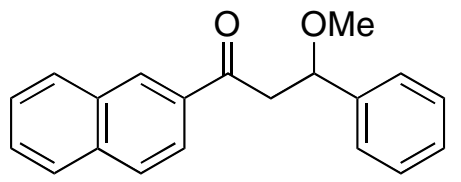

3-Methoxy-1-(2-naphthyl)-3-phenylpropan-1-one (13) The title compound was prepared according to the General Procedure, using 2-acetonaphthone (170 $\mathrm{mg}$, $1.00 \mathrm{mmol})$ as the enolate precursor and benzaldehyde dimethyl acetal $(210 \mu \mathrm{L}$, $213 \mathrm{mg}, 1.40 \mathrm{mmol})$ as the acetal. The product was isolated as a white powder (96\% yield): mp: $87-89{ }^{\circ} \mathrm{C}$; IR (film) 3056, 2928, 1681, 1624, 1467, 1448, 1349, 1273, 1173, 1088, 813, 746 $\mathrm{cm}^{-1} ;{ }^{\mathrm{H}} \mathrm{H}$ NMR $\left(300 \mathrm{MHz}, \mathrm{CDCl}_{3}\right) \delta 8.46(\mathrm{bs}, 1 \mathrm{H}), 8.05(\mathrm{dd}, J=1.8,8.8 \mathrm{~Hz}, 1 \mathrm{H}), 7.99-7.86(\mathrm{~m}, 3 \mathrm{H}), 7.65-7.52$ $(\mathrm{m}, 2 \mathrm{H}), 7.50-7.37(\mathrm{~m}, 4 \mathrm{H}), 7.36-7.29(\mathrm{~m}, 1 \mathrm{H}), 4.97(\mathrm{dd}, J=4.3,8.5 \mathrm{~Hz}, 1 \mathrm{H}), 3.76(\mathrm{dd}, J=8.2,16.2 \mathrm{~Hz}, 1 \mathrm{H})$, $3.29(\mathrm{~s}, 3 \mathrm{H}), 3.23(\mathrm{dd}, J=4.3,16.4 \mathrm{~Hz}, 1 \mathrm{H}) ;{ }^{13} \mathrm{C} \mathrm{NMR}\left(75 \mathrm{MHz}, \mathrm{CDCl}_{3}\right) \delta 197.6,141.5,135.6,134.6,132.5$, 130.1, 129.6, 128.6, 128.5, 128.4, 127.9, 127.8, 126.7, 126.6, 124.0, 79.71, 57.0, 47.3; HRMS (ESI): Exact mass calcd for $\mathrm{C}_{20} \mathrm{H}_{18} \mathrm{O}_{2} \mathrm{Na}[\mathrm{M}+\mathrm{Na}]^{+}, 313.1199$. Found 313.1194.

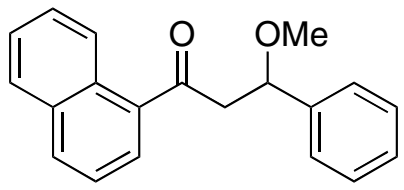

3-Methoxy-1-(1-naphthyl)-3-phenylpropan-1-one (14) The title compound was prepared according to the General Procedure, using 1-acetonaphthone $(152 \mu \mathrm{L}, 170$ $\mathrm{mg}, 1.00 \mathrm{mmol})$ as the enolate precursor and benzaldehyde dimethyl acetal $(210 \mu \mathrm{L}$, $213 \mathrm{mg}, 1.40 \mathrm{mmol})$ as the acetal. The product was isolated as a pale yellow oil $(94 \%$ yield): IR (film) 3056, 2932, 2816, 1670, 1503, 1449, 1348, 1223, 1091, 948, 784, $703 \mathrm{~cm}^{-1} ;{ }^{1} \mathrm{H}$ NMR $\left(300 \mathrm{MHz}, \mathrm{CDCl}_{3}\right) \delta 8.57-8.51(\mathrm{~m}, 1 \mathrm{H}), 7.99(\mathrm{~d}, J=8.2 \mathrm{~Hz}, 1 \mathrm{H}), 7.92-7.82(\mathrm{~m}, 2 \mathrm{H}), 7.63-$ $7.47(\mathrm{~m}, 3 \mathrm{H}), 7.45-7.29(\mathrm{~m}, 5 \mathrm{H}), 4.93(\mathrm{dd}, J=4.2,8.8 \mathrm{~Hz}, 1 \mathrm{H}), 3.64(\mathrm{dd}, J=9.0,15.8 \mathrm{~Hz}, 1 \mathrm{H}), 3.25(\mathrm{~s}, 3 \mathrm{H})$, $3.21(\mathrm{dd}, J=4.4,15.8 \mathrm{~Hz}, 1 \mathrm{H}) ;{ }^{13} \mathrm{C} \mathrm{NMR}\left(75 \mathrm{MHz}, \mathrm{CDCl}_{3}\right) \delta 202.0,141.2,136.5,133.9,132.5,130.1,128.6$, 128.3, 127.9, 127.8, 127.5, 126.7, 127.4, 125.8, 124.3, 80.3, 56.9, 50.9; HRMS (ESI): Exact mass calcd for $\mathrm{C}_{20} \mathrm{H}_{18} \mathrm{O}_{2} \mathrm{Na}[\mathrm{M}+\mathrm{Na}]^{+}, 313.1199$. Found 313.1184 .

(6) For a synthesis of product 11, including NMR data, see: Murata, S.; Suzuki, M.; Noyori, R. Tetrahedron 1988, 44, $4259-4275$. (7) For a synthesis of product 12, including NMR data, see: Wada, M.; Takeichi, E.; Matsumoto, T. Bull. Chem. Soc. Jpn. 1991, 64, 990-994. 


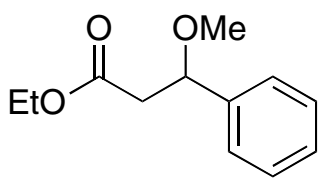

Ethyl 3-methoxy-3-phenylpropanoate (15) The title compound was prepared according to the General Procedure, using ethyl acetate $(98 \mu \mathrm{L}, 88 \mathrm{mg}, 1.00 \mathrm{mmol})$ as the enolate precursor and benzaldehyde dimethyl acetal $(165 \mu \mathrm{L}, 167 \mathrm{mg}, 1.10 \mathrm{mmol})$ as the acetal, except: TESOTf $(317 \mu \mathrm{L}, 371 \mathrm{mg}, 1.40 \mathrm{mmol})$ was used instead of TMSOTf, and the reaction was stirred $24 \mathrm{~h}$. The product was isolated as a colorless oil (81\% yield): IR (film) 2985, 2930, 2876, 2822, 1736, 1448, 1372, 1301, 1263, 1187, 1149, 1095, $758 \mathrm{~cm}^{-1} ;{ }^{1} \mathrm{H}$ NMR $\left(500 \mathrm{MHz}, \mathrm{CDCl}_{3}\right) \delta 7.40-7.29(\mathrm{~m}$, $5 \mathrm{H}), 4.65(\mathrm{dd}, J=5.0,9.4 \mathrm{~Hz}, 1 \mathrm{H}), 4.22-4.11(\mathrm{~m}, 2 \mathrm{H}), 3.25(\mathrm{~s}, 3 \mathrm{H}), 2.83(\mathrm{dd}, J=9.0,15.4 \mathrm{~Hz}, 1 \mathrm{H}), 2.59(\mathrm{dd}, J$ $=4.5,15.3 \mathrm{~Hz}, 1 \mathrm{H}), 1.26(\mathrm{t}, J=7.1 \mathrm{~Hz}, 3 \mathrm{H}) ;{ }^{13} \mathrm{C} \mathrm{NMR}\left(125 \mathrm{MHz}, \mathrm{CDCl}_{3}\right) \delta 171.0,140.7,128.6,128.0,126.6$, 80.1, 660.5, 56.8, 43.6, 14.2; HRMS (ESI): Exact mass calcd for $\mathrm{C}_{12} \mathrm{H}_{16} \mathrm{O}_{3} \mathrm{Na}[\mathrm{M}+\mathrm{Na}]^{+}, 231.0992$. Found 231.0998.<smiles>CCOC(=O)CC(OC)c1ccccc1</smiles>

Isopropyl 3-methoxy-3-phenylpropanoate (16) The title compound was prepared according to the General Procedure, using isopropyl acetate $(117 \mu \mathrm{L}, 102 \mathrm{mg}, 1.00$ $\mathrm{mmol})$ as the enolate precursor and benzaldehyde dimethyl acetal $(165 \mu \mathrm{L}, 167 \mathrm{mg}, 1.10$ $\mathrm{mmol})$ as the acetal, except: TESOTf $(317 \mu \mathrm{L}, 371 \mathrm{mg}, 1.40 \mathrm{mmol})$ was used instead of TMSOTf, and the reaction was stirred $24 \mathrm{~h}$. The product was isolated as a yellow oil (76\% yield): IR (film) 2977, 2929, 2819, 1729, 1447, 1370, 1267, 1190, 1157, 1099, 765, $699 \mathrm{~cm}^{-1} ;{ }^{1} \mathrm{H}$ NMR $\left(500 \mathrm{MHz} \mathrm{CDCl}_{3}\right)$ $\delta$ 7.40-7.29 (m, 5H), $5.04(\mathrm{sp}, J=6.3 \mathrm{~Hz}, 1 \mathrm{H}), 4.64(\mathrm{dd}, J=5.0,9.1 \mathrm{~Hz}, 1 \mathrm{H}), 4.22-4.11(\mathrm{~m}, 2 \mathrm{H}), 3.25(\mathrm{~s}, 3 \mathrm{H})$, $2.80(\mathrm{dd}, J=8.9,15.2 \mathrm{~Hz}, 1 \mathrm{H}), 2.58(\mathrm{dd}, J=5.0,15.2 \mathrm{~Hz}, 1 \mathrm{H}), 1.25(\mathrm{~d}, J=6.4 \mathrm{~Hz}, 3 \mathrm{H}), 1.20(\mathrm{~d}, J=6.2 \mathrm{~Hz}$, $3 \mathrm{H}) ;{ }^{13} \mathrm{C} \mathrm{NMR}\left(125 \mathrm{MHz}, \mathrm{CDCl}_{3}\right) \delta 170.4,140.7,128.5,128.0,126.7,80.2,67.9,56.8,43.8,21.8,21.7$; HRMS (ESI): Exact mass calcd for $\mathrm{C}_{13} \mathrm{H}_{18} \mathrm{O}_{3} \mathrm{Na}[\mathrm{M}+\mathrm{Na}]^{+}, 245.1148$. Found 245.1148.

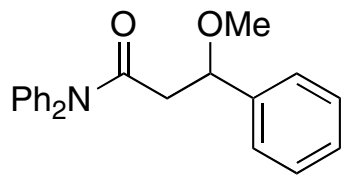

3-Methoxy-3,N,N-triphenylpropanamide (17) The title compound was prepared according to the General Procedure, using $N, N$-diphenylacetamide $(212 \mathrm{mg}, 1.00 \mathrm{mmol})$ as the enolate precursor and benzaldehyde dimethyl acetal $(210 \mu \mathrm{L}, 213 \mathrm{mg}, 1.40 \mathrm{mmol})$ as the acetal. The product was isolated as an orange-white solid, contaminated with $<5 \%$ starting material $\left(80 \%\right.$ yield): $\mathrm{mp}=106-109^{\circ} \mathrm{C}$; IR (film) $3052,2924,2812,1666,1588,1487,1441,1355$, 1282, 1095, 750, $695 \mathrm{~cm}^{-1} ;{ }^{1} \mathrm{H}$ NMR $\left(500 \mathrm{MHz}, \mathrm{CDCl}_{3}\right) \delta 7.38-7.26(\mathrm{~m}, 10 \mathrm{H}), 7.14(\mathrm{bs}, 5 \mathrm{H}), 4.82(\mathrm{dd}, J=5.6$, $7.8 \mathrm{~Hz}, 1 \mathrm{H}), 4.64(\mathrm{dd}, J=5.0,9.1 \mathrm{~Hz}, 1 \mathrm{H}), 3.27(\mathrm{~s}, 3 \mathrm{H}), 2.85(\mathrm{dd}, J=7.9,15.1 \mathrm{~Hz}, 1 \mathrm{H}), 2.56(\mathrm{dd}, J=5.8,14.9$ $\mathrm{Hz}, 1 \mathrm{H}) ;{ }^{13} \mathrm{C}$ NMR $\left(125 \mathrm{MHz}, \mathrm{CDCl}_{3}\right) \delta 170.3,142.8,141.2,129.6,128.9,128.1,127.8,126.8,126.6,80.8$, 56.9, 43.6, 23.9; HRMS (ESI): Exact mass calcd for $\mathrm{C}_{13} \mathrm{H}_{18} \mathrm{O}_{3} \mathrm{Na}[\mathrm{M}+\mathrm{Na}]^{+}, 354.1465$. Found 354.1470.

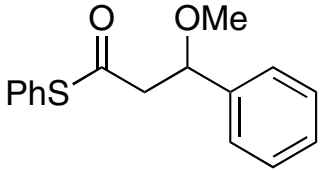

$S$-Phenyl 3-methoxy-3-phenylpropanethioate (18) The title compound was prepared according to the General Procedure, using $S$-phenyl thioacetate $(135 \mu \mathrm{L}, 152 \mathrm{mg}, 1.00$ $\mathrm{mmol})$ as the enolate precursor and benzaldehyde dimethyl acetal $(210 \mu \mathrm{L}, 213 \mathrm{mg}, 1.40$ mmol) as the acetal, except: higher loadings of TMSOTf $(271 \mu \mathrm{L}, 333 \mathrm{mg}, 1.50 \mathrm{mmol})$ and $i$ - $\operatorname{Pr}_{2} \mathrm{NEt}(261 \mu \mathrm{L}, 194 \mathrm{mg}, 1.50 \mathrm{mmol})$ were necessary to achieve higher conversion. The product was isolated as a pale yellow oil (94\% yield): IR (film) 3055, 2979, 2930, 2811, 1698, 1464, 1437, 1095, 1052, 965, $737 \mathrm{~cm}^{-1} ;{ }^{1} \mathrm{H}$ NMR $\left(300 \mathrm{MHz}, \mathrm{CDCl}_{3}\right) \delta 7.46-7.30(\mathrm{~m}, 10 \mathrm{H}), 4.72(\mathrm{dd}, J=4.3,8.8 \mathrm{~Hz}, 1 \mathrm{H}), 3.27(\mathrm{~s}, 3 \mathrm{H}), 3.14$ $(\mathrm{dd}, J=8.9,15.2 \mathrm{~Hz}, 1 \mathrm{H}), 2.88(\mathrm{dd}, J=4.4,15.2 \mathrm{~Hz}, 1 \mathrm{H}) ;{ }^{13} \mathrm{C} \mathrm{NMR}\left(75 \mathrm{MHz}, \mathrm{CDCl}_{3}\right) \delta 194.9,140.3,134.5$, 129.4, 129.2, 128.7, 128.1, 127.7, 126.6, 79.9, 57.0, 51.9; HRMS (ESI): Exact mass calcd for $\mathrm{C}_{17} \mathrm{H}_{18} \mathrm{O}_{2} \mathrm{SNa}$ $[\mathrm{M}+\mathrm{Na}]^{+}, 309.0920$. Found 309.0921.

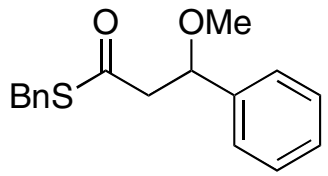

S-Benzyl 3-methoxy-3-phenylpropanethioate (19) The title compound was prepared according to the General Procedure, using $S$-benzyl thioacetate $(150 \mu \mathrm{L}, 166 \mathrm{mg}, 1.00$ 
$\mathrm{mmol})$ as the enolate precursor and benzaldehyde dimethyl acetal $(210 \mu \mathrm{L}, 213 \mathrm{mg}, 1.40 \mathrm{mmol})$ as the acetal, except: higher loadings of TMSOTf $(271 \mu \mathrm{L}, 333 \mathrm{mg}, 1.50 \mathrm{mmol})$ and $i$-Pr ${ }_{2} \mathrm{NEt}(261 \mu \mathrm{L}, 194 \mathrm{mg}, 1.50 \mathrm{mmol})$ were necessary to achieve higher conversion. The product was isolated as a colorless oil ( $84 \%$ yield): IR (film) 3027, 2928, 2814, 1690, 1501, 1449, 1104, 1052, 976, 764, 735, 707, 613, $556 \mathrm{~cm}^{-1}$; ${ }^{1} \mathrm{H}$ NMR $(500 \mathrm{MHz}$, $\left.\mathrm{CDCl}_{3}\right) \delta 7.41-7.23(\mathrm{~m}, 10 \mathrm{H}), 4.71(\mathrm{dd}, J=4.3,9.1 \mathrm{~Hz}, 1 \mathrm{H}), 4.20(\mathrm{~d}, J=13.8 \mathrm{~Hz}, 1 \mathrm{H}), 4.14(\mathrm{~d}, J=13.9 \mathrm{~Hz}$, $1 \mathrm{H}), 3.24(\mathrm{~s}, 3 \mathrm{H}), 3.10(\mathrm{dd}, J=9.1,14.8 \mathrm{~Hz}, 1 \mathrm{H}), 2.81(\mathrm{dd}, J=4.4,14.9 \mathrm{~Hz}, 1 \mathrm{H}) ;{ }^{13} \mathrm{C} \mathrm{NMR}\left(125 \mathrm{MHz} \mathrm{CDCl}_{3}\right)$ $\delta 196.1,140.3,137.4,128.8,128.6,128.5,128.1,127.2,126.6,80.1,56.9,52.1,33.3$; HRMS (ESI): Exact mass calcd for $\mathrm{C}_{17} \mathrm{H}_{18} \mathrm{O}_{2} \mathrm{SNa}[\mathrm{M}+\mathrm{Na}]^{+}$, 309.0920. Found 309.0921.

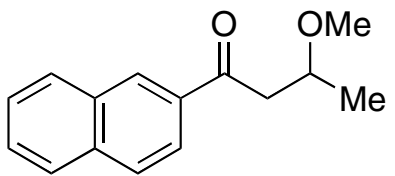

3-Methoxy-1-(2-naphthyl)-3-butan-1-one (20) The title compound was prepared according to the General Procedure, using 2-acetonaphthone $(170 \mathrm{mg}, 1.00 \mathrm{mmol})$ as the enolate precursor and acetaldehyde dimethyl acetal $(148 \mu \mathrm{L}, 126 \mathrm{mg}, 1.40 \mathrm{mmol})$ as the acetal, except: higher loading of TMSOTf $(220 \mu \mathrm{L}, 270 \mathrm{mg}, 1.22 \mathrm{mmol})$ was necessary to achieve higher conversion. Note: this reaction is extremely sensitive to mole ratio of TMSOTf. Use of 1.20 equiv of TMSOTf resulted in lowered conversion (33-65\%), and use of 1.22 equiv TMSOTf resulted in 5-10\% elimination of the methoxy group. ${ }^{8}$ The product was isolated as a pale yellow oil (95\% yield): IR (film) 2973, 1672, 1468, 1369, 1310, 1194, 1129, 1100, 826, $744 \mathrm{~cm}^{-1} ;{ }^{1} \mathrm{H}$ NMR $\left(500 \mathrm{MHz}, \mathrm{CDCl}_{3}\right) \delta 8.50$ (bs, $1 \mathrm{H}), 8.06(\mathrm{dd}, J=1.9,8.8 \mathrm{~Hz}, 1 \mathrm{H}), 7.99(\mathrm{~d}, J=8.0 \mathrm{~Hz}, 1 \mathrm{H}), 7.93-7.88(\mathrm{~m}, 2 \mathrm{H}), 7.64-7.55(\mathrm{~m}, 2 \mathrm{H}), 4.08$ (ap sx, $J=6.5 \mathrm{~Hz}, 1 \mathrm{H}), 3.50(\mathrm{dd}, J=6.7,16.1 \mathrm{~Hz}, 1 \mathrm{H}), 3.39(\mathrm{~s}, 3 \mathrm{H}), 3.06(\mathrm{dd}, J=6.3,16.3 \mathrm{~Hz}, 1 \mathrm{H}), 1.33(\mathrm{~d}, J=6.2$ $\mathrm{Hz}, 3 \mathrm{H}) ;{ }^{13} \mathrm{C}$ NMR $\left(125 \mathrm{MHz}, \mathrm{CDCl}_{3}\right) \delta 198.6,135.6,134.7,132.6,130.0,129.6,128.5,128.4,127.8,126.8$, 123.9, 73.7, 56.5, 45.6, 19.7; HRMS (ESI): Exact mass calcd for $\mathrm{C}_{15} \mathrm{H}_{16} \mathrm{O}_{2} \mathrm{Na}[\mathrm{M}+\mathrm{Na}]^{+}, 251.1043$. Found 251.1034.

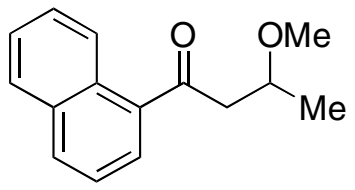

3-Methoxy-1-(1-naphthyl)-3-butan-1-one (21) The title compound was prepared according to the General Procedure, using 1-acetonaphthone (152 $\mu \mathrm{L}, 170 \mathrm{mg}, 1.00$ $\mathrm{mmol})$ as the enolate precursor and acetaldehyde dimethyl acetal $(148 \mu \mathrm{L}, 126 \mathrm{mg}, 1.40$ mmol) as the acetal, except: higher loading of TMSOTf $(220 \mu \mathrm{L}, 270 \mathrm{mg}, 1.22 \mathrm{mmol})$ was necessary to achieve higher conversion. Note: this reaction is extremely sensitive to mole ratio of TMSOTf. Use of 1.20 equiv of TMSOTf resulted in lowered conversion (33-65\%), and use of 1.22 equiv TMSOTf resulted in 5-10\% elimination of the methoxy group. The product was isolated as a yellow oil (94\% yield): IR (film) 2980, 2826, 1683, 1511, 1372, 1297, 1233, 1177, 1139, 1087, 798, $783 \mathrm{~cm}^{-1} ;{ }^{1} \mathrm{H} \mathrm{NMR}$ $\left(500 \mathrm{MHz}, \mathrm{CDCl}_{3}\right) \delta 8.59(\mathrm{~d}, J=8.8 \mathrm{~Hz}, 1 \mathrm{H}), 8.00(\mathrm{~d}, J=8.0 \mathrm{~Hz}, 1 \mathrm{H}), 7.90(\mathrm{~m}, 2 \mathrm{H}), 7.63-7.59(\mathrm{~m}, 1 \mathrm{H}), 7.57-$ $7.50(\mathrm{~m}, 2 \mathrm{H}), 4.09-4.01(\mathrm{~m}, 1 \mathrm{H}), 3.42(\mathrm{dd}, J=7.1,15.6 \mathrm{~Hz}, 1 \mathrm{H}), 3.35(\mathrm{~s}, 3 \mathrm{H}), 3.03(\mathrm{dd}, J=5.7,15.7 \mathrm{~Hz}, 1 \mathrm{H})$, $1.31(\mathrm{~d}, J=6.2,3 \mathrm{H}) ;{ }^{13} \mathrm{C}$ NMR $\left(125 \mathrm{MHz}, \mathrm{CDCl}_{3}\right) \delta 202.9,136.6,134.0,132.5,130.1,128.4,127.8,127.5$, 126.4, 125.8, 124.4, 74.0, 56.5, 49.3, 19.5; HRMS (ESI): Exact mass calcd for $\mathrm{C}_{15} \mathrm{H}_{16} \mathrm{O}_{2} \mathrm{Na}[\mathrm{M}+\mathrm{Na}]^{+}$, 251.1043. Found 251.1055.<smiles>COC(C)CC(=O)Sc1ccccc1</smiles>

S-Phenyl 3-methoxybutanethioate (24) The title compound was prepared according to the General Procedure, using $S$-phenyl thioacetate $(135 \mu \mathrm{L}, 152 \mathrm{mg}, 1.00 \mathrm{mmol})$ as the enolate precursor and acetaldehyde dimethyl acetal $(148 \mu \mathrm{L}, 126 \mathrm{mg}, 1.40 \mathrm{mmol})$ as the acetal, except: higher loadings of TMSOTf $(271 \mu \mathrm{L}, 333 \mathrm{mg}, 1.50 \mathrm{mmol})$ and $i$ - $\operatorname{Pr}_{2} \mathrm{NEt}(261 \mu \mathrm{L}, 194 \mathrm{mg}, 1.50 \mathrm{mmol})$ were necessary to achieve higher conversion. The product was isolated as a pale yellow oil (81\% yield): IR (film) 2974, 2925, 2822, 1703, 1470, 1437, 1361, 1084, 976, $731 \mathrm{~cm}^{-1} ;{ }^{1} \mathrm{H} \mathrm{NMR}\left(300 \mathrm{MHz}, \mathrm{CDCl}_{3}\right) \delta 7.43$ (bs, $5 \mathrm{H}), 3.86(\mathrm{ap} \mathrm{dq}, J=6.1,7.0 \mathrm{~Hz}, 1 \mathrm{H}), 3.38(\mathrm{~s}, 3 \mathrm{H}), 2.97(\mathrm{dd}, J=7.0,14.9 \mathrm{~Hz}, 1 \mathrm{H}), 2.70(\mathrm{dd}, J=5.6,14.9 \mathrm{~Hz}$, $1 \mathrm{H}), 1.26(\mathrm{~d}, J=6.3 \mathrm{~Hz}, 3 \mathrm{H}) ;{ }^{13} \mathrm{C} \mathrm{NMR}\left(75 \mathrm{MHz}, \mathrm{CDCl}_{3}\right) \delta 195.4,134.4,129.4,129.2,127.8,73.7,56.5,50.3$, 19.3; HRMS (ESI): Exact mass calcd for $\mathrm{C}_{11} \mathrm{H}_{14} \mathrm{O}_{2} \mathrm{SNa}[\mathrm{M}+\mathrm{Na}]^{+}, 233.0607$. Found 233.0596.

(8) This product is known to undergo elimination of the $\beta$-methoxy group to yield the $\alpha, \beta$-unsaturated ketone under Lewis acidic conditions. See: Ramirez, F.; Rubin, M. B. J. Am. Chem. Soc. 1955, 77, 2905-2907. 
O OMe $S$-Benzyl 3-methoxybutanethioate (25) The title compound was prepared according to the General Procedure, using $S$-benzyl thioacetate $(150 \mu \mathrm{L}, 166 \mathrm{mg}, 1.00 \mathrm{mmol})$ as the enolate precursor and acetaldehyde dimethyl acetal $(148 \mu \mathrm{L}, 126 \mathrm{mg}, 1.40 \mathrm{mmol})$ as the acetal, except: higher loadings of TMSOTf $(362 \mu \mathrm{L}, 445 \mathrm{mg}, 2.00 \mathrm{mmol})$ and $i$ - $\operatorname{Pr}_{2} \mathrm{NEt}(348 \mu \mathrm{L}, 258 \mathrm{mg}, 2.00 \mathrm{mmol})$ were necessary to achieve higher conversion. The product was isolated as a colorless oil (71\% yield): IR (film) 2974, 2930, 2822, 1681, 1491, 1453, 1372, 1138, 1084, 992, 758, $699 \mathrm{~cm}^{-1} ;{ }^{1} \mathrm{H}$ NMR $\left(500 \mathrm{MHz}_{2} \mathrm{CDCl}_{3}\right)$ $\delta$ 7.34-7.29 (m, 4H), 7.27-7.23 (m, 1H), $4.16($ ap dd, $J=13.8,18.3 \mathrm{~Hz}, 2 \mathrm{H}), 3.87-3.80(\mathrm{~m}, 1 \mathrm{H}), 3.34(\mathrm{~s}, 3 \mathrm{H})$, $2.88(\mathrm{dd}, J=6.9,14.6 \mathrm{~Hz}, 1 \mathrm{H}), 2.62(\mathrm{dd}, J=5.7,14.9 \mathrm{~Hz}, 1 \mathrm{H}), 1.22(\mathrm{~d}, J=6.1 \mathrm{~Hz}, 3 \mathrm{H}) ;{ }^{13} \mathrm{C} \mathrm{NMR}(125 \mathrm{MHz}$, $\left.\mathrm{CDCl}_{3}\right) \delta 196.6,137.5,128.8,128.6,127.3,73.8,56.5,50.5,33.3,19.3$; HRMS (ESI): Exact mass calcd for $\mathrm{C}_{12} \mathrm{H}_{16} \mathrm{O}_{2} \mathrm{SNa}[\mathrm{M}+\mathrm{Na}]^{+}, 247.0763$. Found 247.0761.

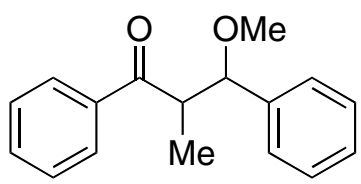

3-Methoxy-2-methyl-1,3-diphenylpropan-1-one (26) The title compound $^{9}$ was prepared according to the General Procedure, using propiophenone $(133 \mu \mathrm{L}, 134 \mathrm{mg}$, $1.00 \mathrm{mmol})$ as the enolate precursor and benzaldehyde dimethyl acetal $(210 \mu \mathrm{L}, 213 \mathrm{mg}$, $1.40 \mathrm{mmol}$ ) as the acetal. The product (mixture of diastereomers) was isolated as a colorless oil (82\% yield, 3.1:1 syn:anti): IR (film) 2979, 2932, 2819, 1670, 1588, 1445, 1212, 1173, 1091, 963, $750,695 \mathrm{~cm}^{-1} ;{ }^{1} \mathrm{H}$ NMR $\left(500 \mathrm{MHz}, \mathrm{CDCl}_{3}\right)$ syn isomer: $\delta 7.78-7.72(\mathrm{~m}, 2 \mathrm{H}), 7.54-7.14(\mathrm{~m}, 8 \mathrm{H}), 4.49(\mathrm{~d}, J=$ $8.1 \mathrm{~Hz}, 1 \mathrm{H}), 3.85(\mathrm{dq}, J=6.8,7.9 \mathrm{~Hz}, 1 \mathrm{H}), 3.23(\mathrm{~s}, 3 \mathrm{H}), 1.38(\mathrm{~d}, J=6.8 \mathrm{~Hz}, 3 \mathrm{H}) ;$ anti isomer: $\delta 8.10-8.04(\mathrm{~m}$, $2 \mathrm{H}), 7.63-7.22(\mathrm{~m}, 8 \mathrm{H}), 4.50(\mathrm{~d}, J=9.4 \mathrm{~Hz}, 1 \mathrm{H}), 3.81(\mathrm{dq}, J=7.0,9.7 \mathrm{~Hz}, 1 \mathrm{H}), 3.11(\mathrm{~s}, 3 \mathrm{H}), 0.88(\mathrm{~d}, J=6.8$ $\mathrm{Hz}, 3 \mathrm{H}) ;{ }^{13} \mathrm{C} \mathrm{NMR}\left(125 \mathrm{MHz}, \mathrm{CDCl}_{3}\right)$ syn isomer: $\delta 202.5,140.3,137.0,132.7,128.5,128.2,128.1,127.7$, 127.4, 84.7, 56.9, 48.6, 14.5; anti isomer: $\delta 203.7,139.8,127.7,132.9,128.6,128.5,128.4,128.1,127.7,86.4$, 56.8, 47.3, 14.9; HRMS (ESI): Exact mass calcd for $\mathrm{C}_{17} \mathrm{H}_{18} \mathrm{O}_{2} \mathrm{Na}[\mathrm{M}+\mathrm{Na}]^{+}, 277.1199$. Found 277.1206.<smiles>COC(C)C(C)C(=O)c1ccccc1</smiles>

3-Methoxy-2-methyl-1-phenylbutan-1-one (27) The title compound $^{10}$ was prepared according to the General Procedure, using propiophenone $(133 \mu \mathrm{L}, 134 \mathrm{mg}, 1.00 \mathrm{mmol})$ as the enolate precursor and acetaldehyde dimethyl acetal $(148 \mu \mathrm{L}, 126 \mathrm{mg}, 1.40 \mathrm{mmol})$ as the acetal, except: higher loading of TMSOTf $(226 \mu \mathrm{L}, 278 \mathrm{mg}, 1.25 \mathrm{mmol})$ was necessary to achieve higher conversion. The product (mixture of diastereomers) was isolated as a yellow oil (82\% yield, 3.7:1 syn:anti): IR (film) 2984, 2937, 2819, 2356, 1685, 1600, 1458, 1387, 1222, 1094, 962, $702 \mathrm{~cm}^{-1} ;{ }^{1} \mathrm{H} \mathrm{NMR}$ $\left(500 \mathrm{MHz}, \mathrm{CDCl}_{3}\right)$ syn isomer: $\delta$ 7.98-7.95 $(\mathrm{m}, 2 \mathrm{H}), 7.61-7.54(\mathrm{~m}, 1 \mathrm{H}), 7.51-7.46(\mathrm{~m}, 2 \mathrm{H}), 3.67-3.60(\mathrm{~m}, 1 \mathrm{H})$, $3.56(\mathrm{dq}, J=7.0,7.2 \mathrm{~Hz}, 1 \mathrm{H}), 3.36(\mathrm{~s}, 3 \mathrm{H}), 1.29(\mathrm{~d}, J=7.0 \mathrm{~Hz}, 3 \mathrm{H}), 1.16(\mathrm{~d}, J=6.2 \mathrm{~Hz}, 3 \mathrm{H})$; anti isomer: $\delta$ 8.00-7.96 (m, 2H), 7.60-7.54 (m, 1H), 7.51-7.46 (m, 2H), $3.73(\mathrm{dq}, J=6.5,8.0 \mathrm{~Hz}, 1 \mathrm{H}), 3.67-3.60(\mathrm{~m}, 1 \mathrm{H})$, $3.29(\mathrm{~s}, 3 \mathrm{H}), 1.21(\mathrm{~d}, J=6.1 \mathrm{~Hz}, 3 \mathrm{H}), 1.14(\mathrm{~d}, J=6.8 \mathrm{~Hz}, 3 \mathrm{H}) ;{ }^{13} \mathrm{C}$ NMR $\left(125 \mathrm{MHz}, \mathrm{CDCl}_{3}\right)$ syn isomer: $\delta 203.3,137.2,132.9,128.6,128.3,78.6,56.8,47.1,17.3,14.3$; anti isomer: $\delta 203.7,137.5,132.8,128.5$, 128.3, 78.9, 56.8, 46.5, 15.9, 13.3; HRMS (ESI): Exact mass calcd for $\mathrm{C}_{12} \mathrm{H}_{16} \mathrm{O}_{2} \mathrm{Na}[\mathrm{M}+\mathrm{Na}]^{+}, 215.1043$. Found 215.1032.

(9) For a literature synthesis including NMR data and assignment of stereoisomers, see reference 6. (10) Identities of diastereomers assigned in analogy to product $\mathbf{2 6}$. 
Journal of Organic Chemistry

Supporting Information Page 8

${ }^{1} \mathrm{H}$ and ${ }^{13} \mathrm{C}$ NMR Spectra

Product 1
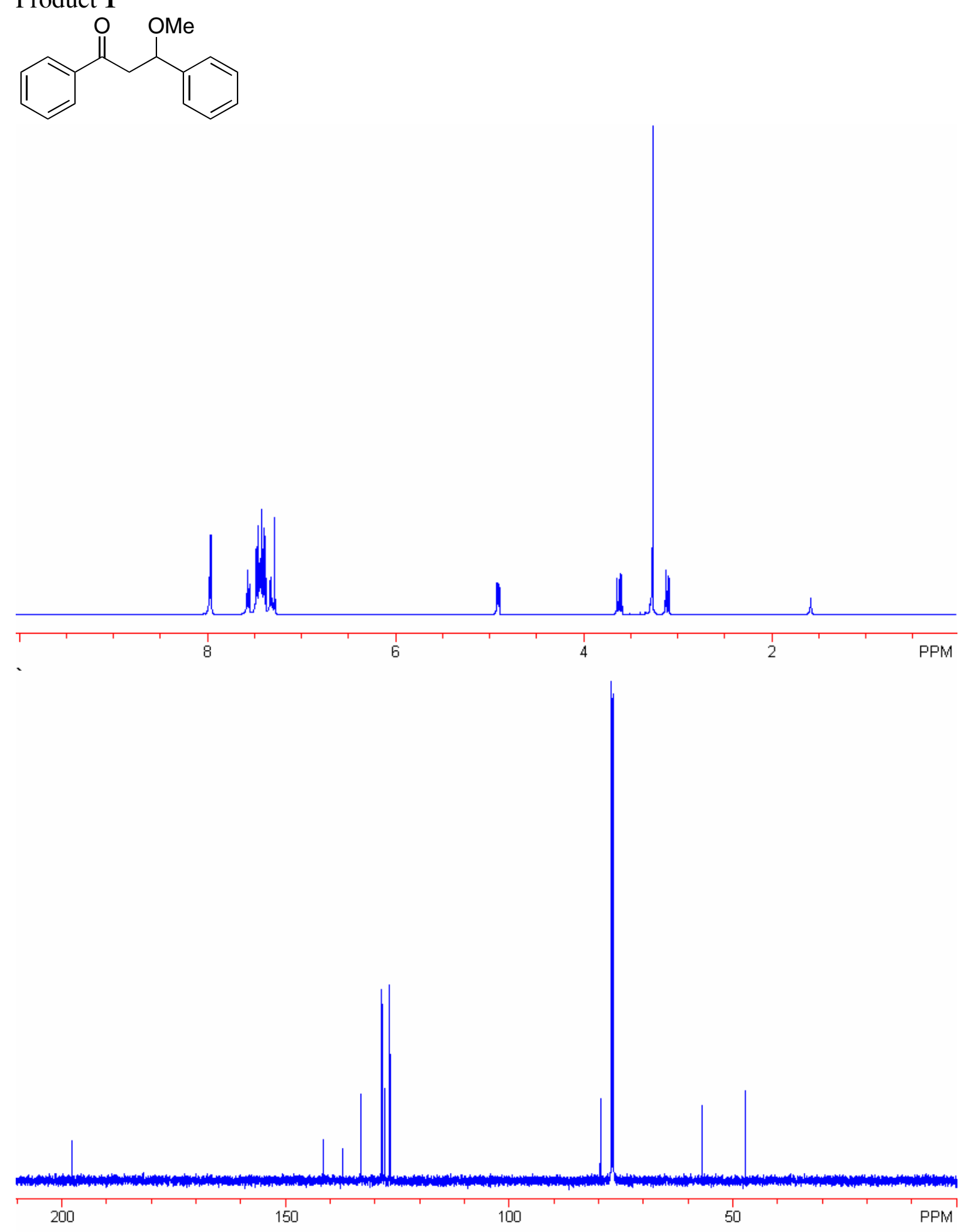
Product 2<smiles>COc1ccc(C(CC(=O)c2ccccc2)OC)cc1</smiles>
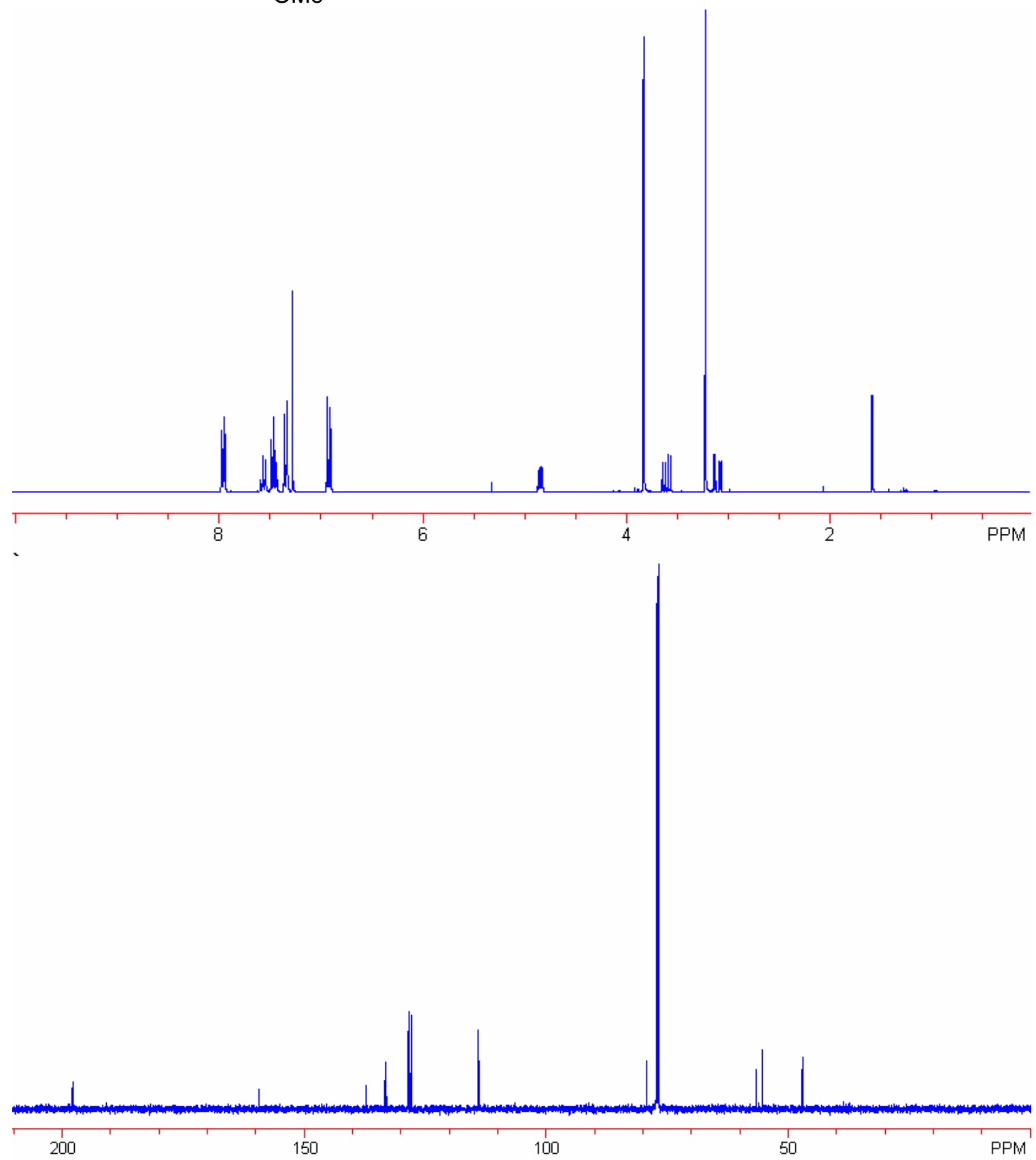
Product 3<smiles>COC(CC(=O)c1ccccc1)c1ccco1</smiles>
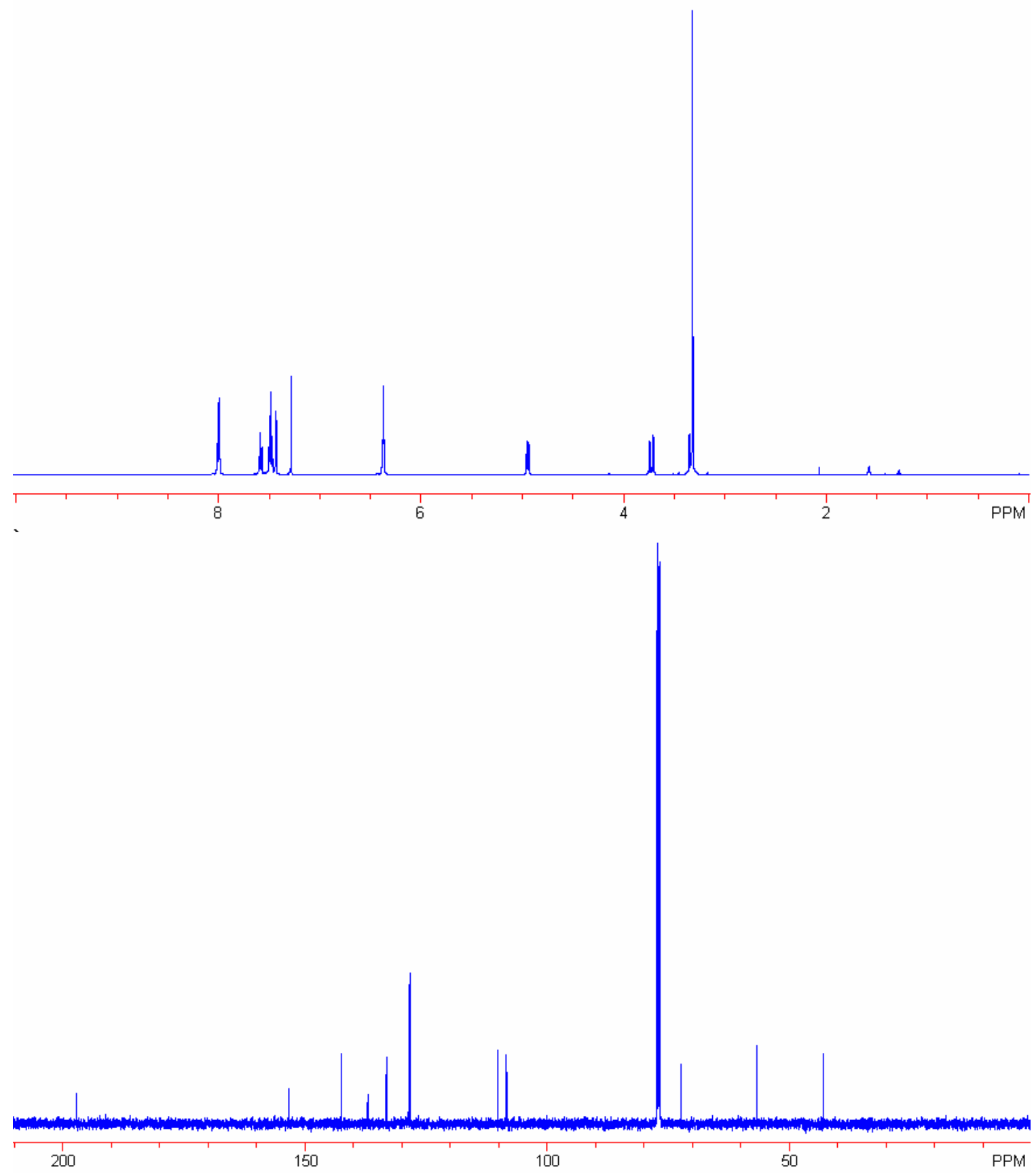
Product 4<smiles>COC(CC(=O)c1ccccc1)c1cccs1</smiles>
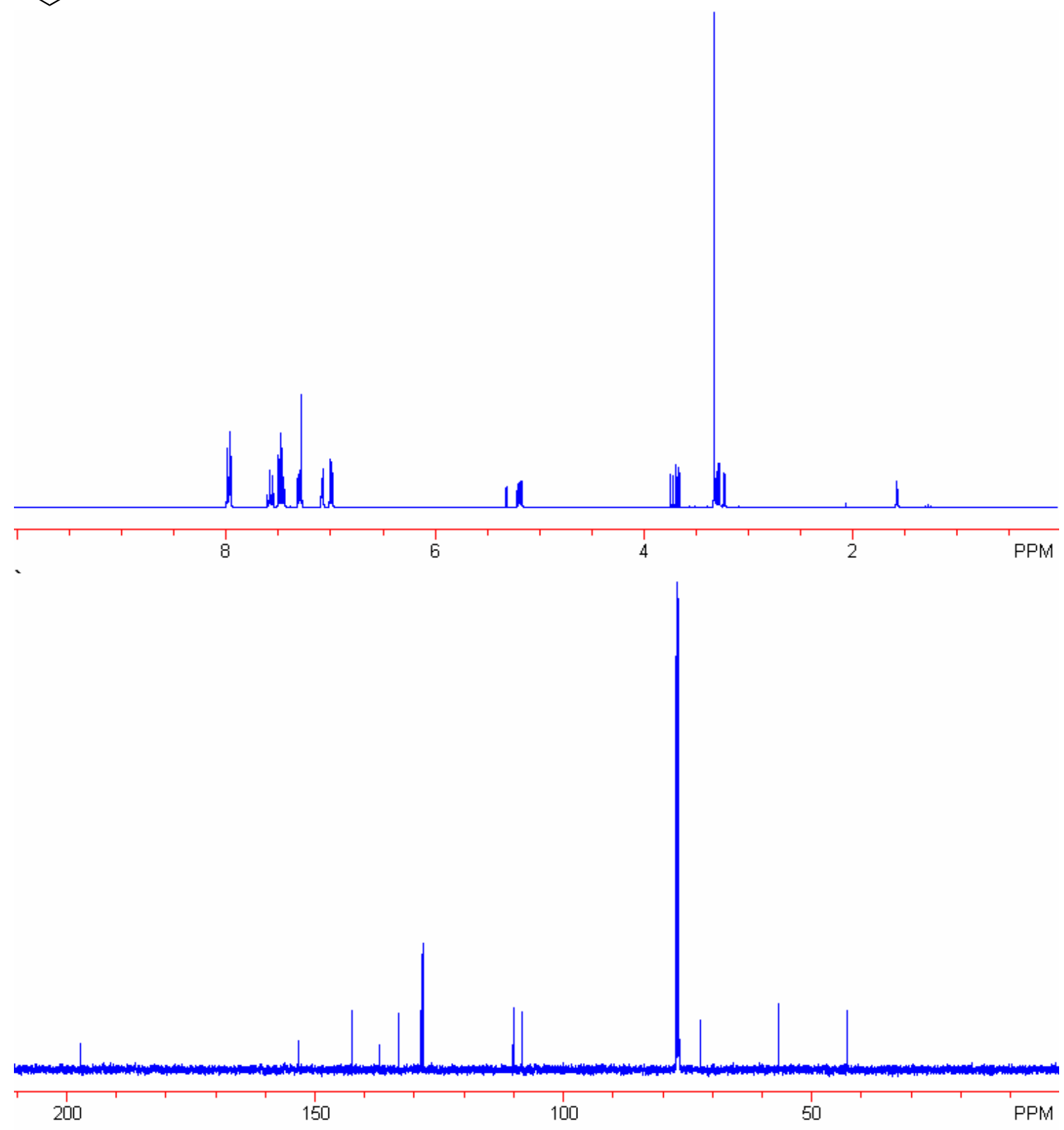
Product 5<smiles>COC(CC(=O)c1ccccc1)c1ccc2ccccc2c1</smiles>
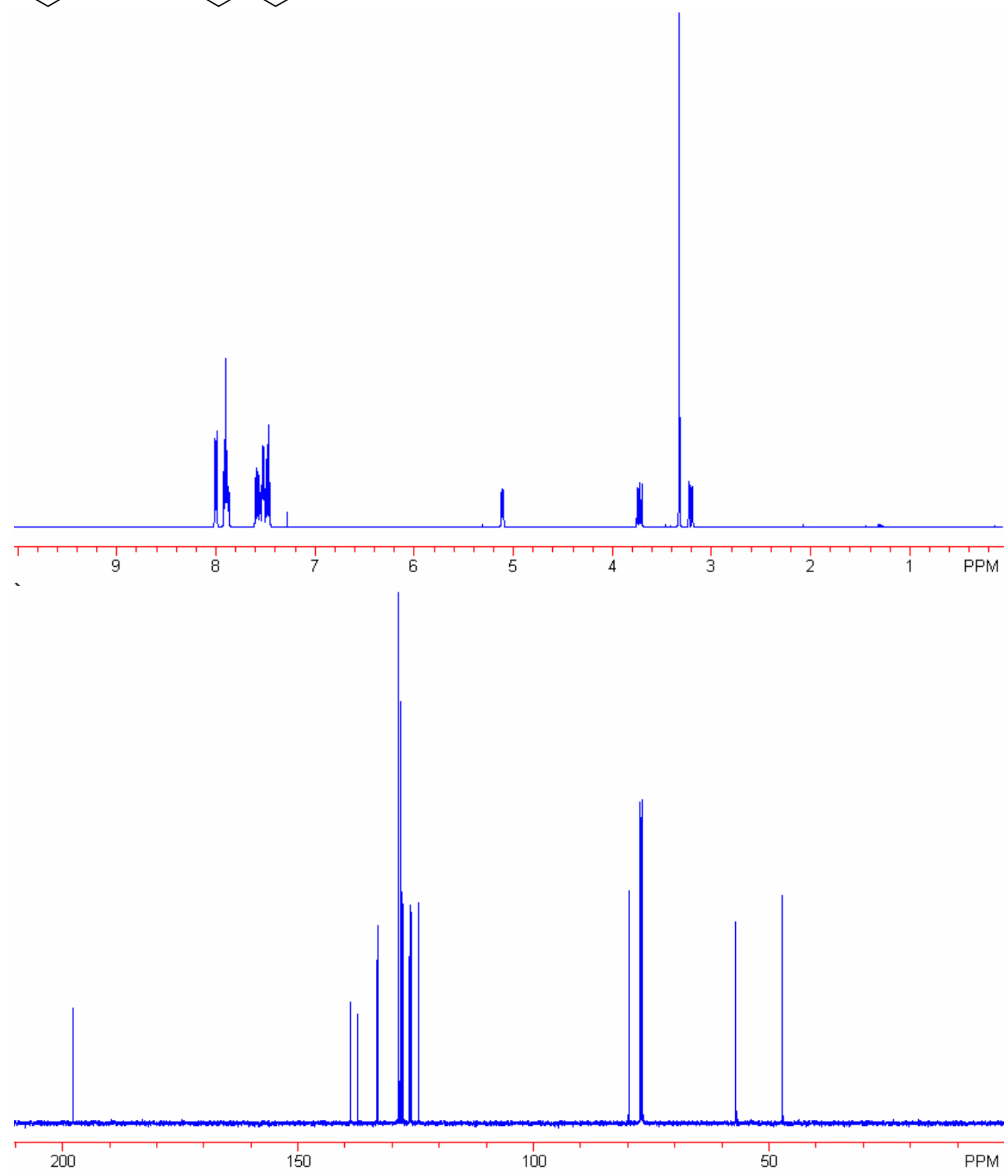
Product 6<smiles>COC(/C=C/c1ccccc1)CC(=O)c1ccccc1</smiles>
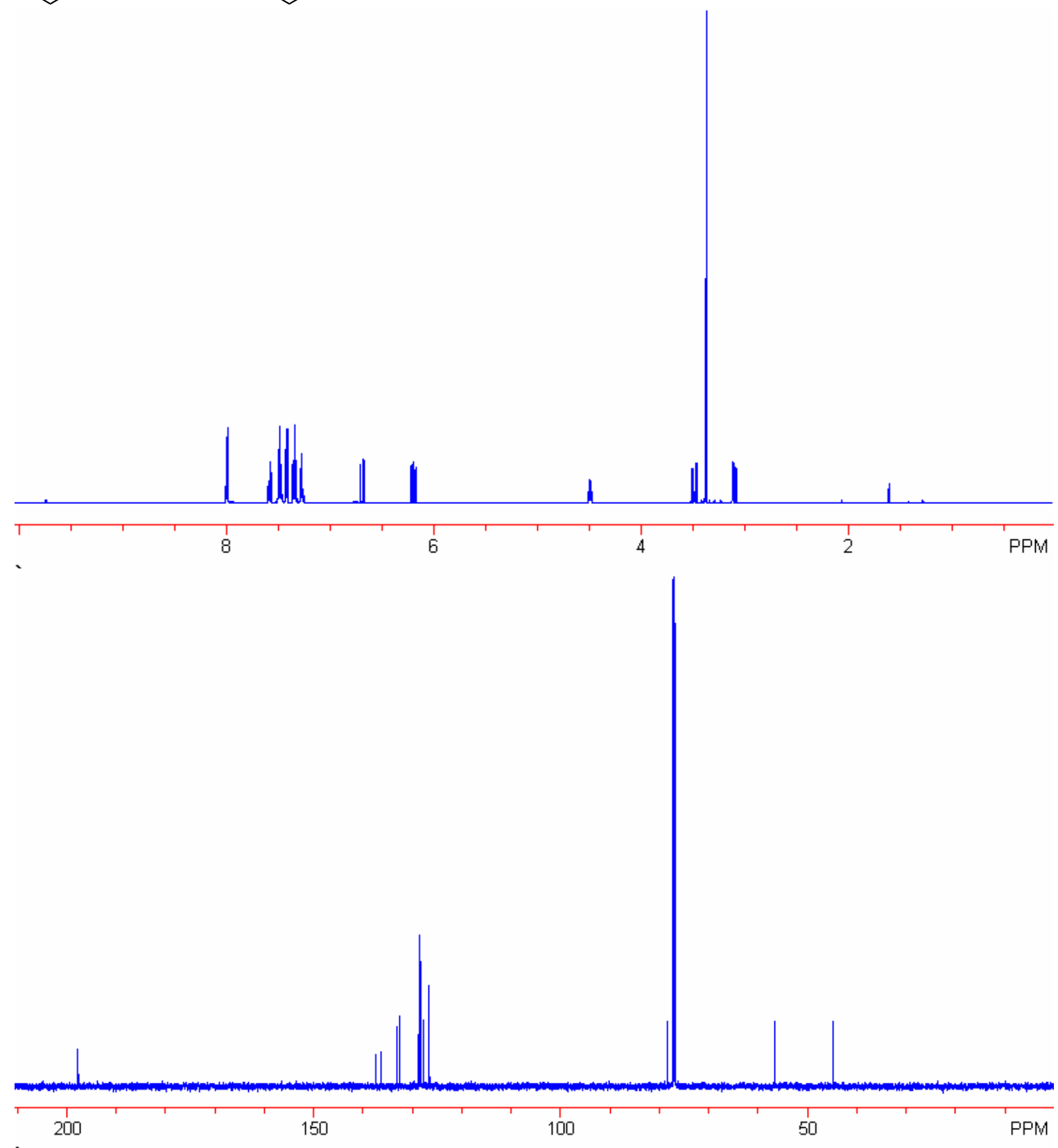
Product 7<smiles>COC(CC(=O)c1ccccc1)C1CCCCC1</smiles>

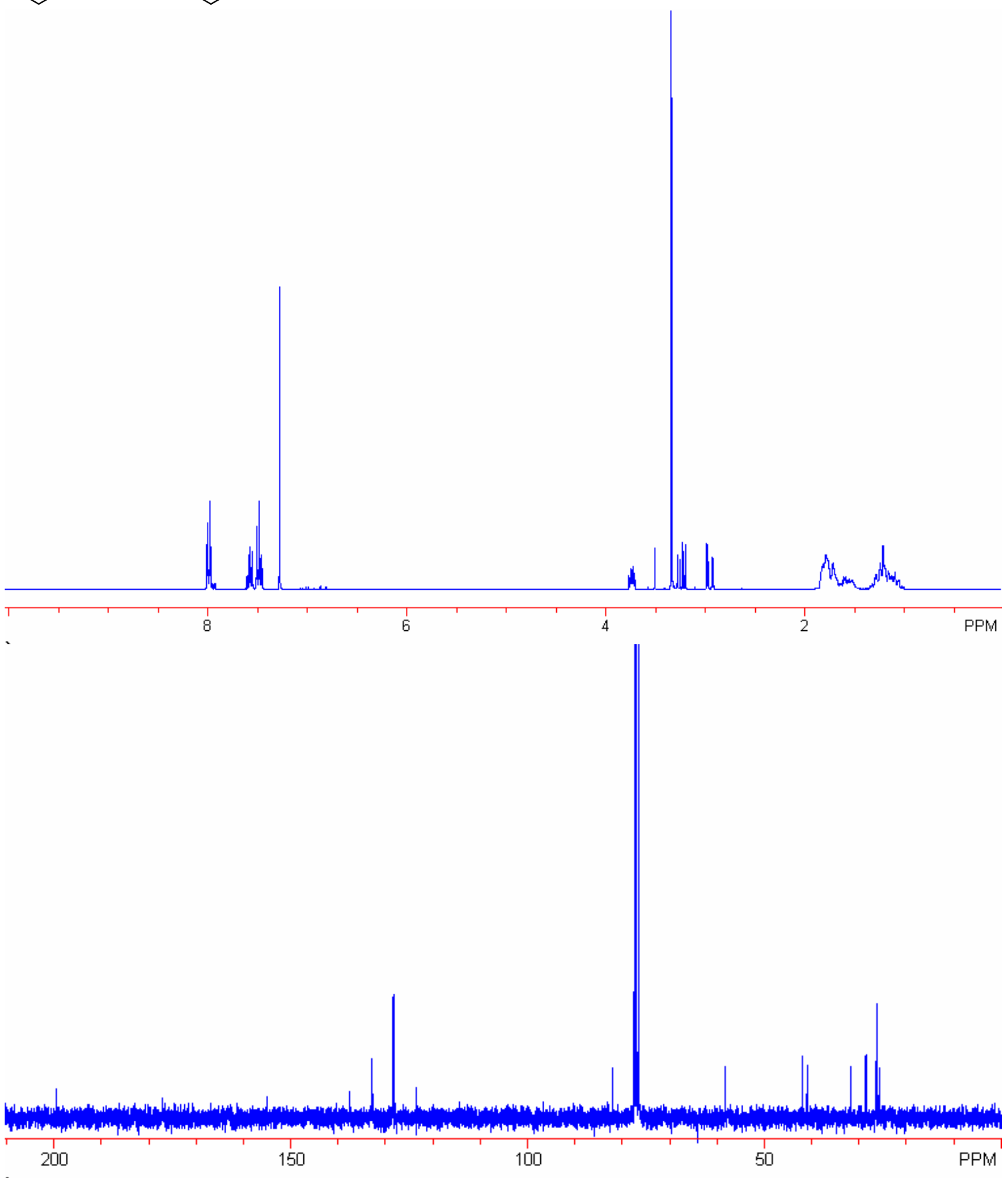




\section{Product 8}<smiles>COC(C)CC(=O)c1ccccc1</smiles>
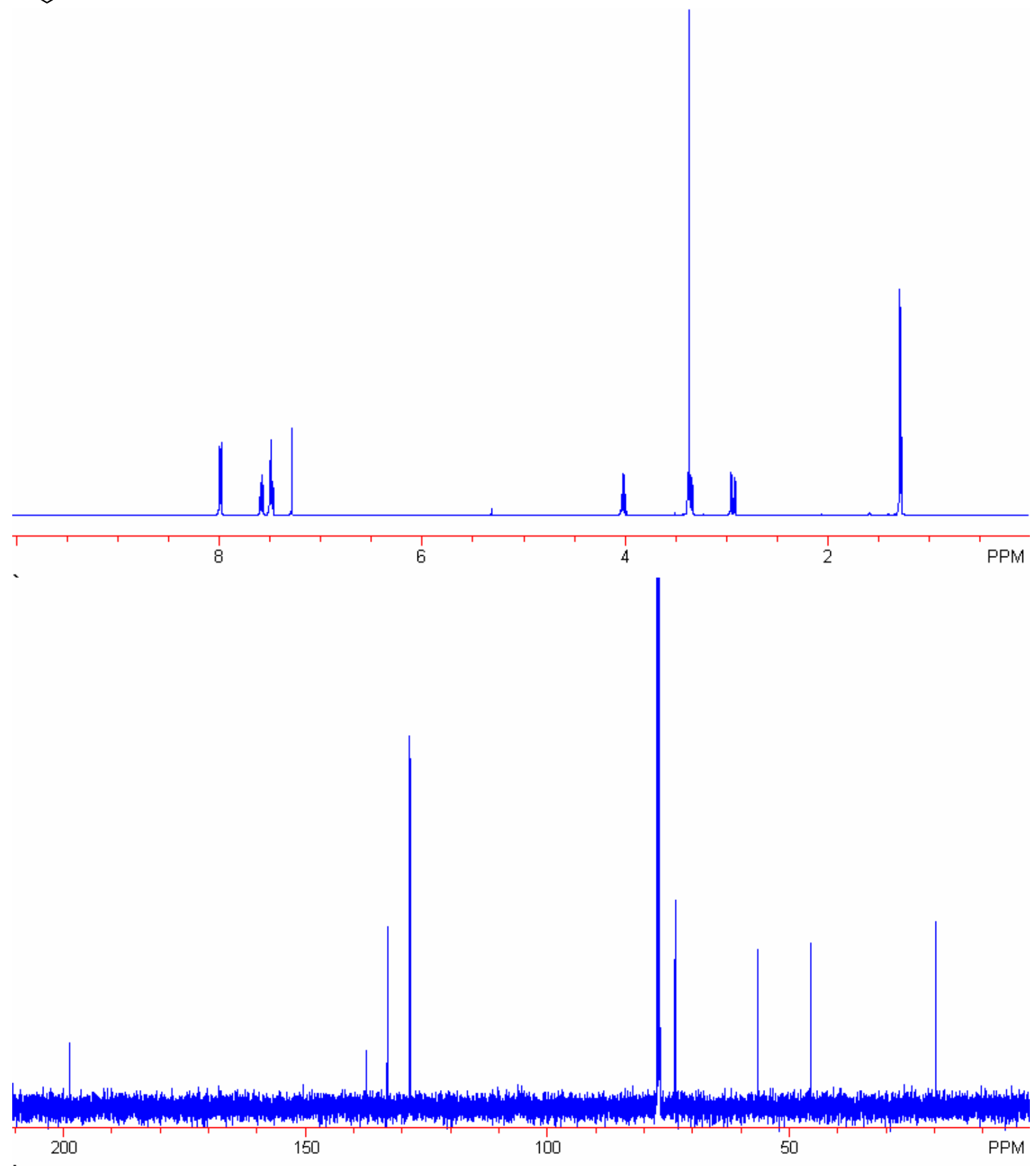
Product 9<smiles>CCC(CC(=O)c1ccccc1)OC</smiles>
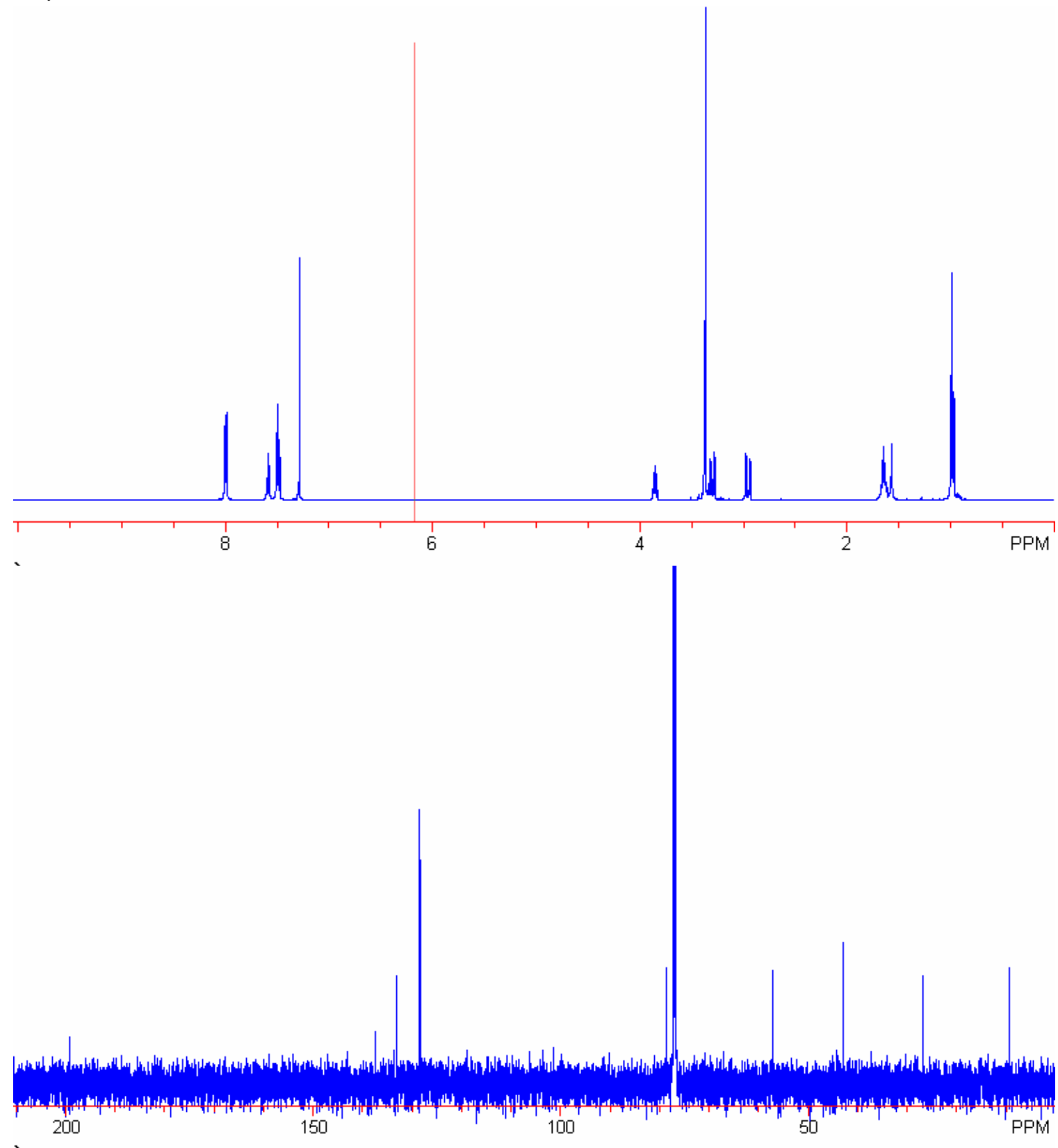


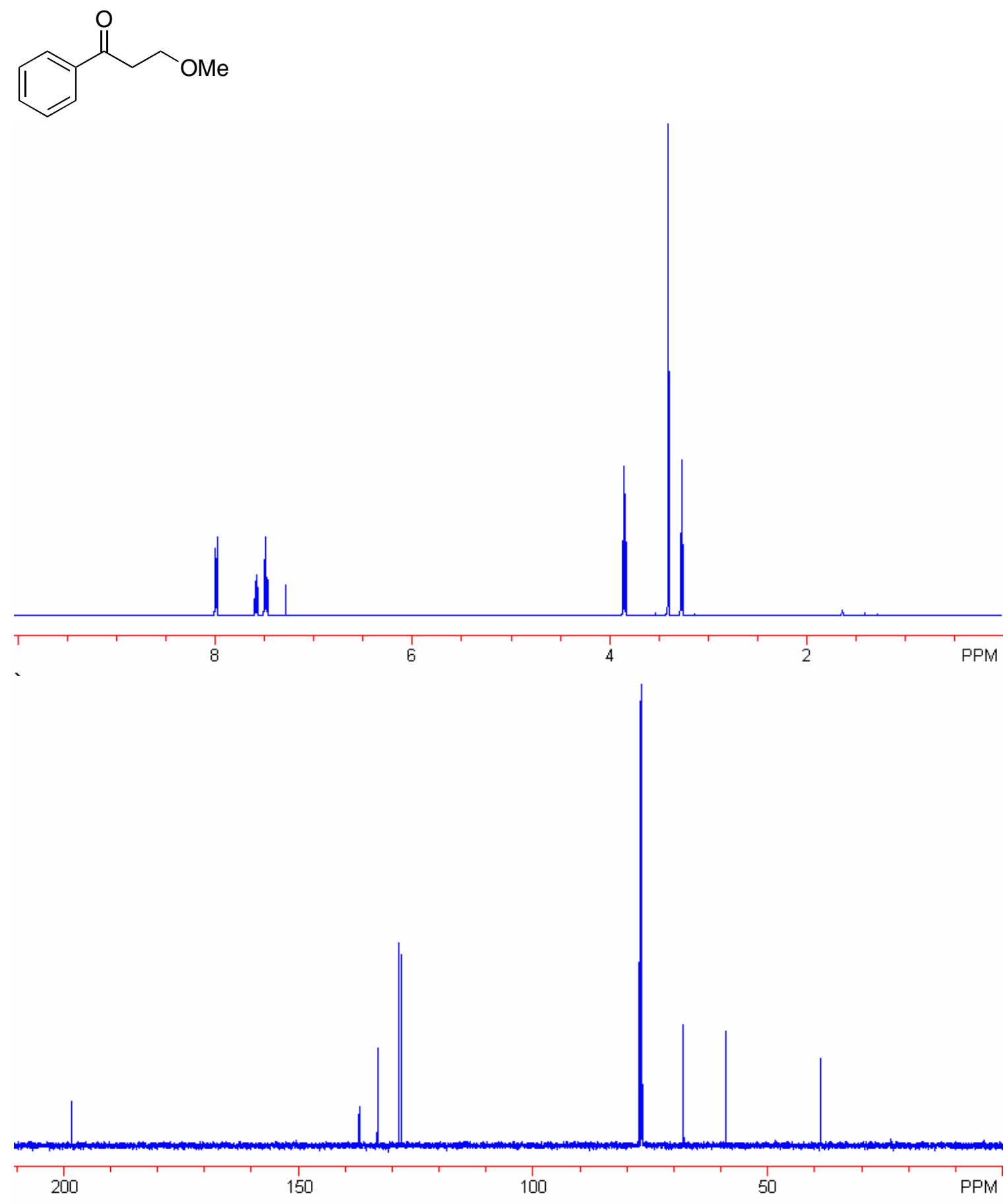

Product 11 
<smiles>O=C(CC1CCCCO1)c1ccccc1</smiles>

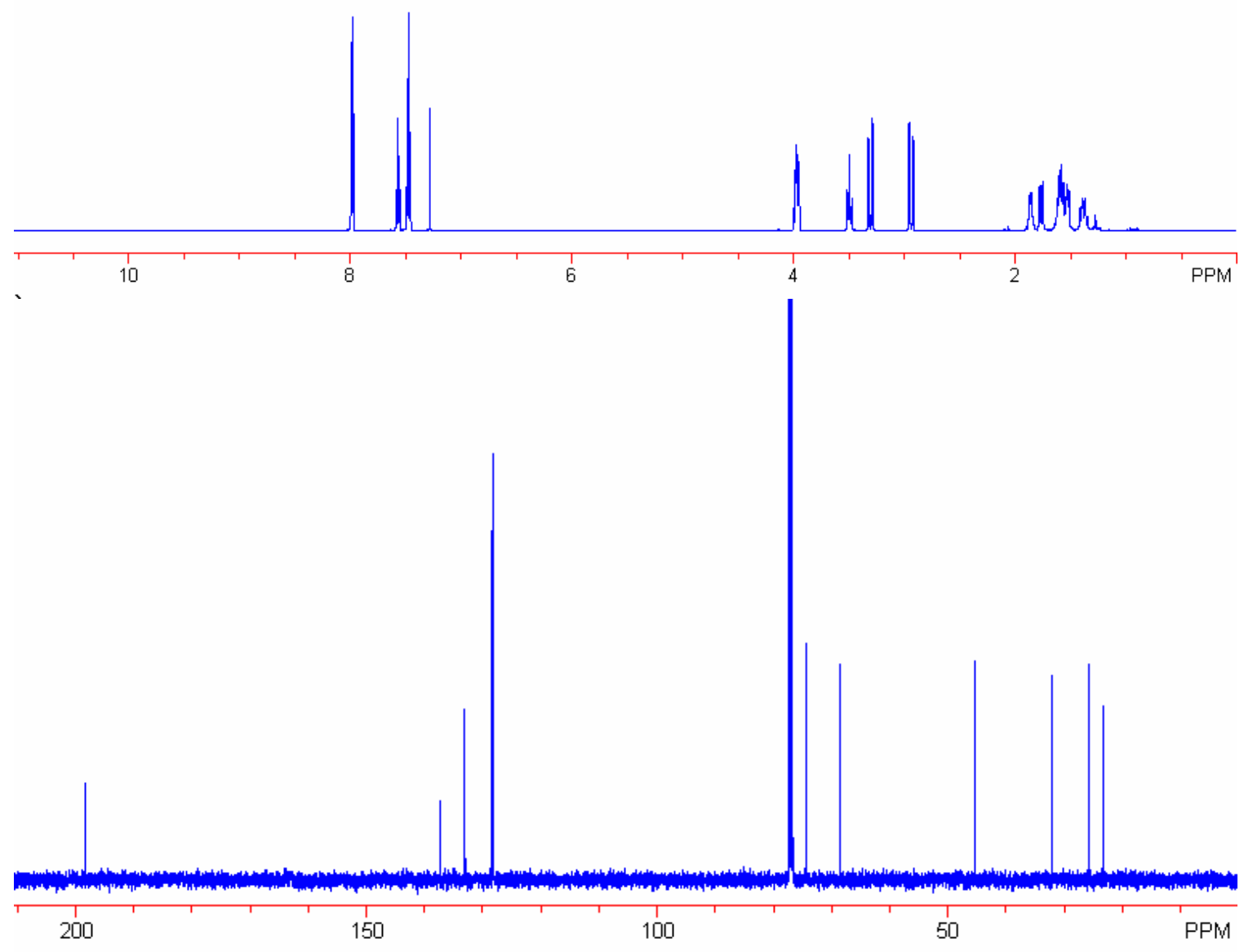

Product 12 


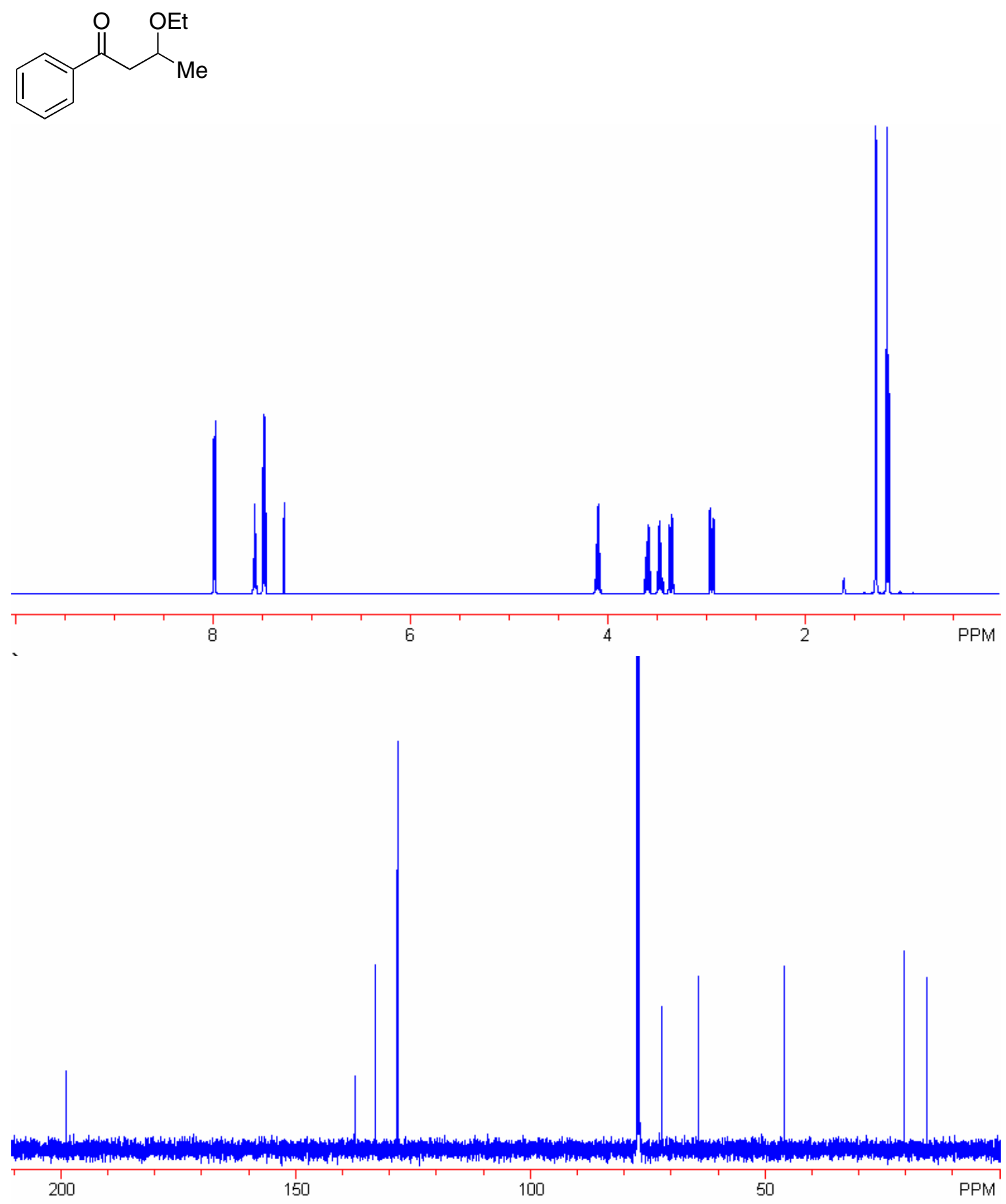

Product 13 


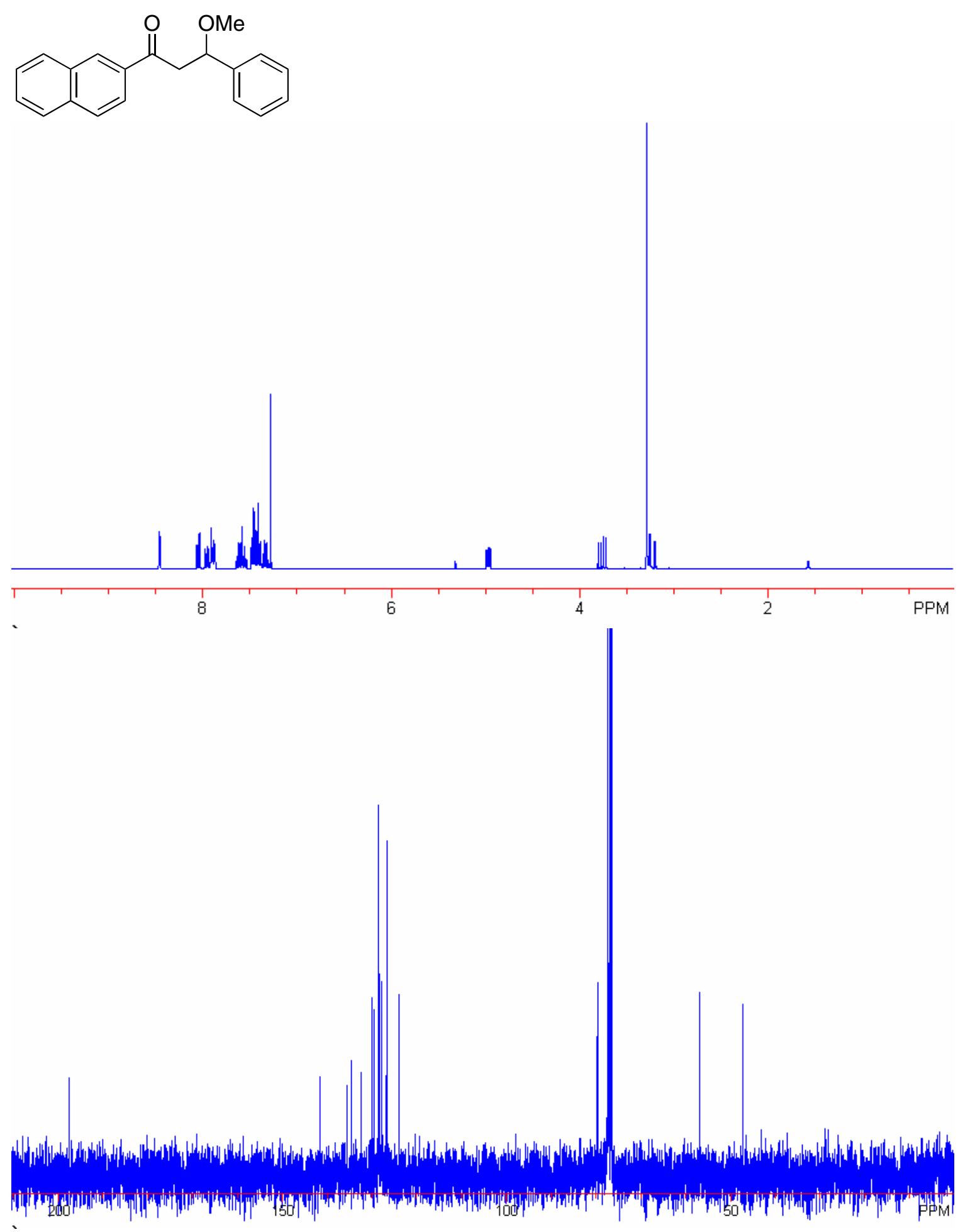

Product 14 
<smiles>COC(CC(=O)c1cccc2ccccc12)c1ccccc1</smiles>
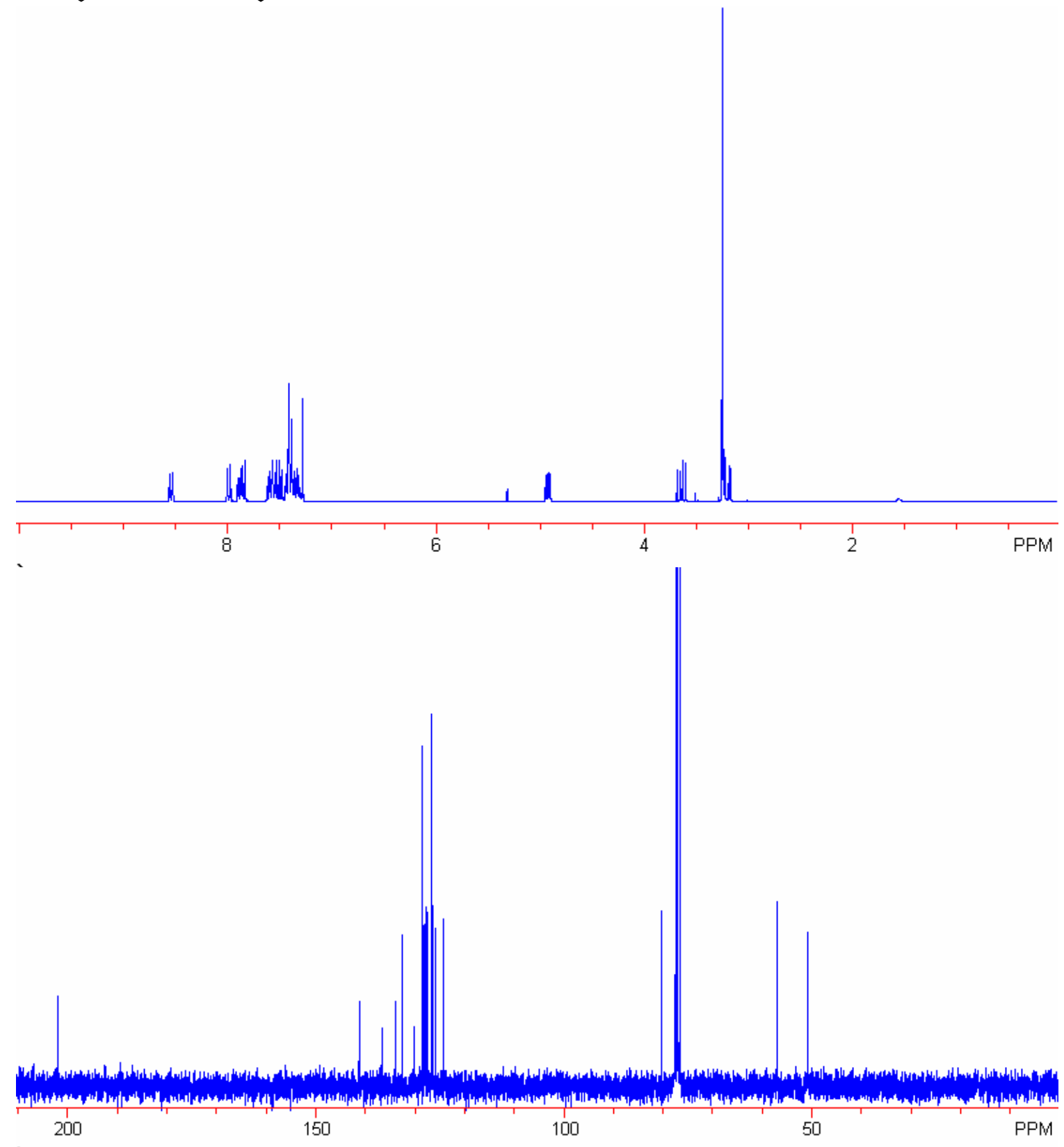

Product 15 
<smiles>CCOC(=O)CC(OC)c1ccccc1</smiles>
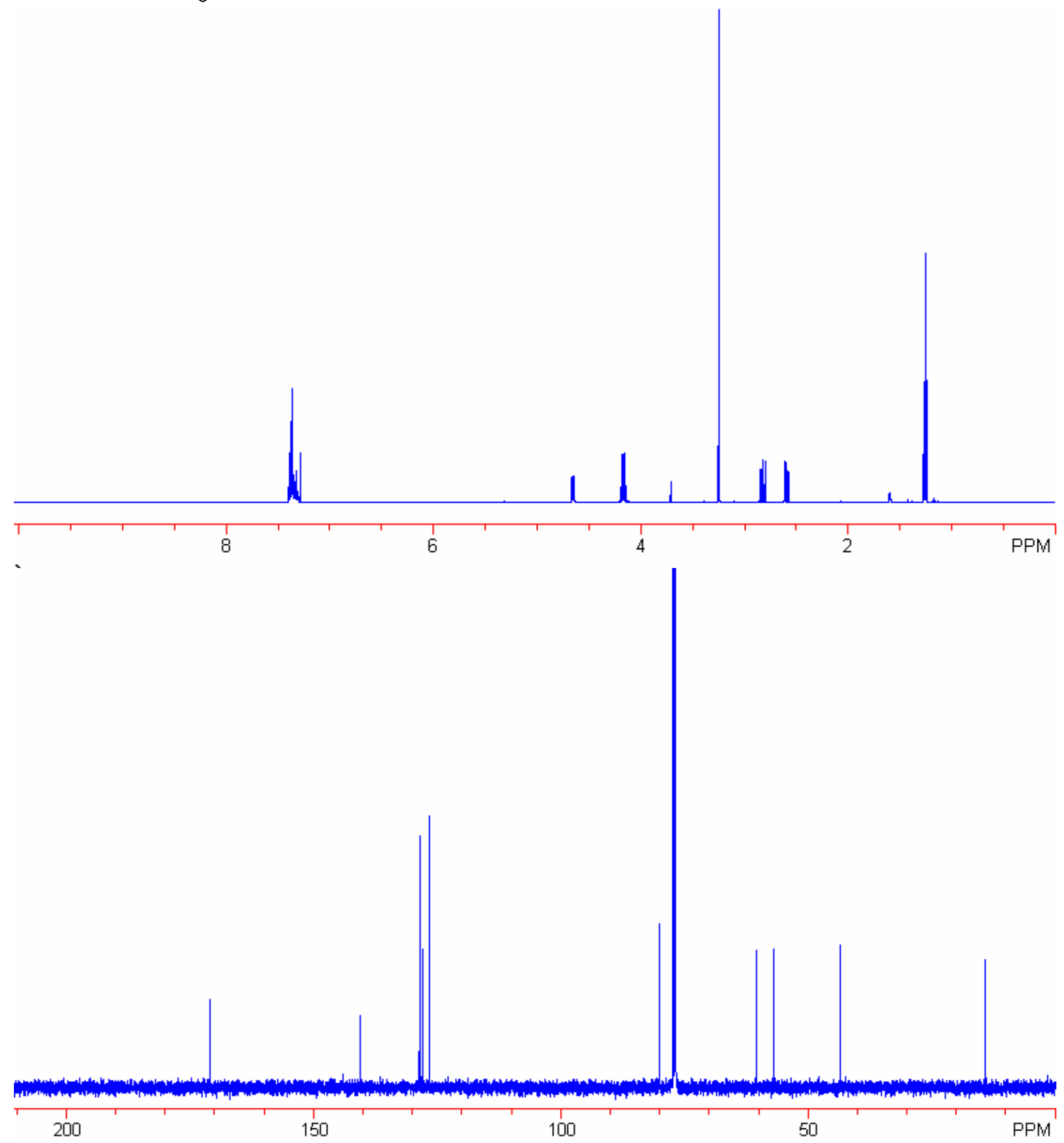

Product 16 
<smiles>COC(CC(=O)O[In])c1ccccc1</smiles>

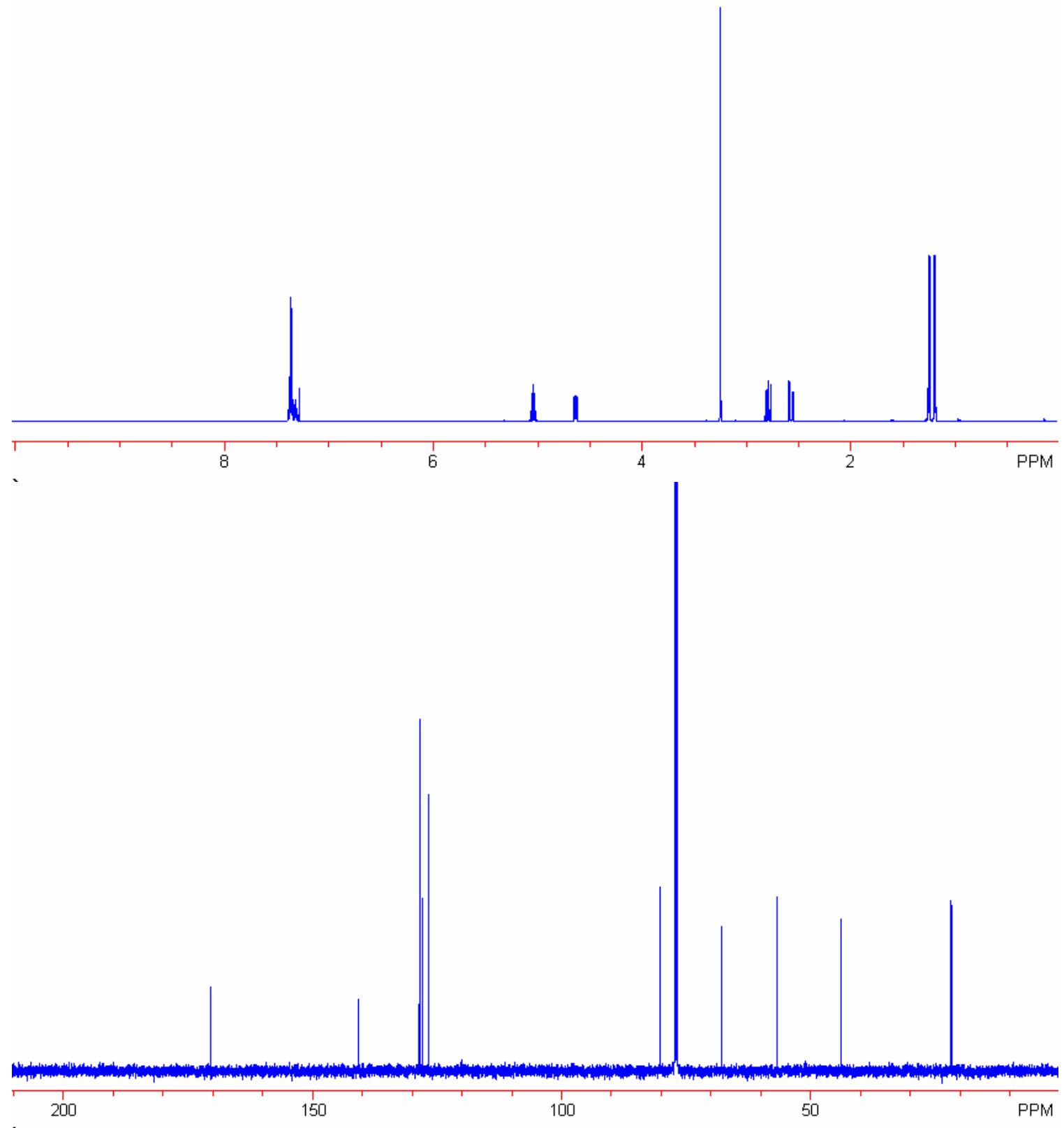

Product 17 

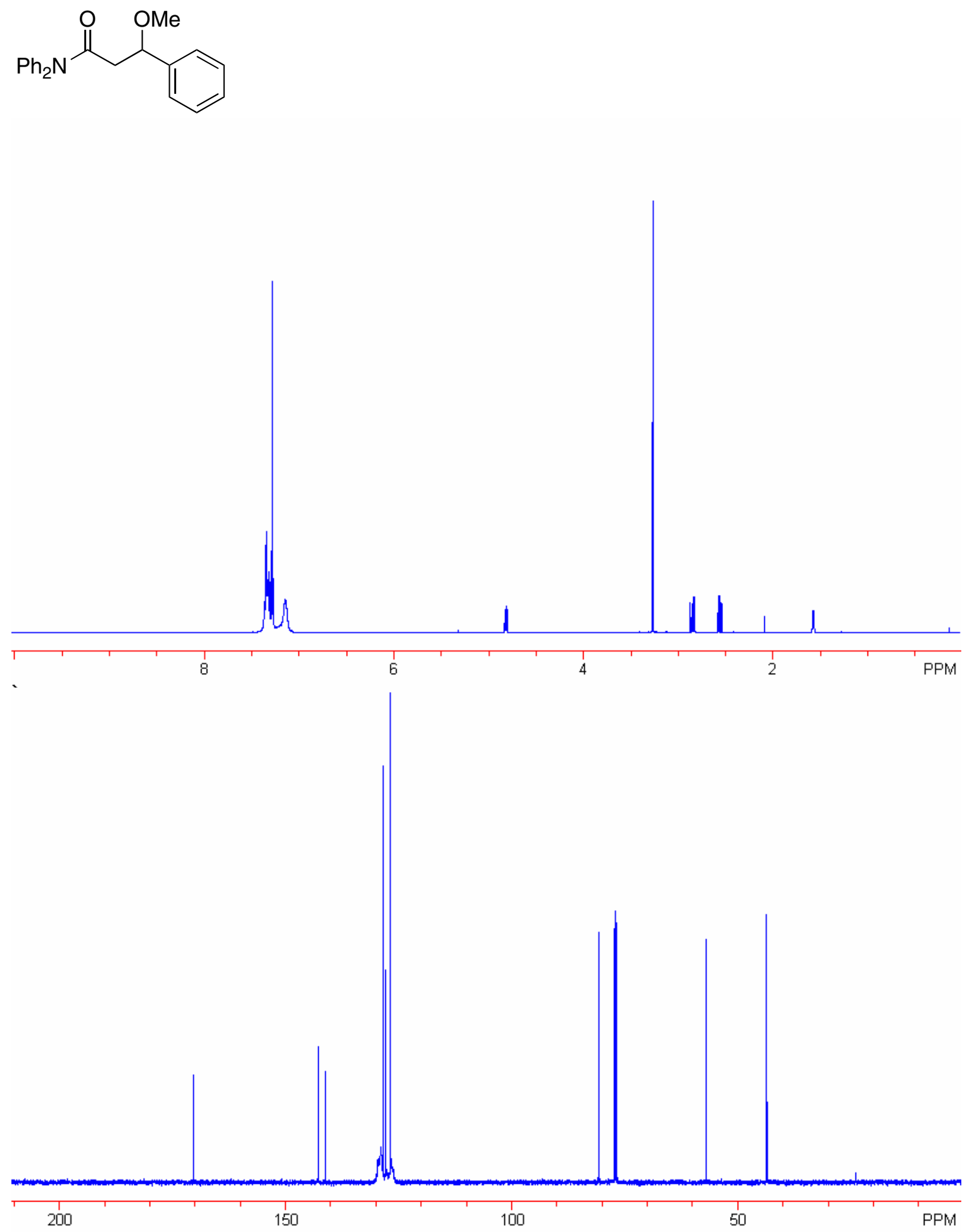

\section{Product 18}




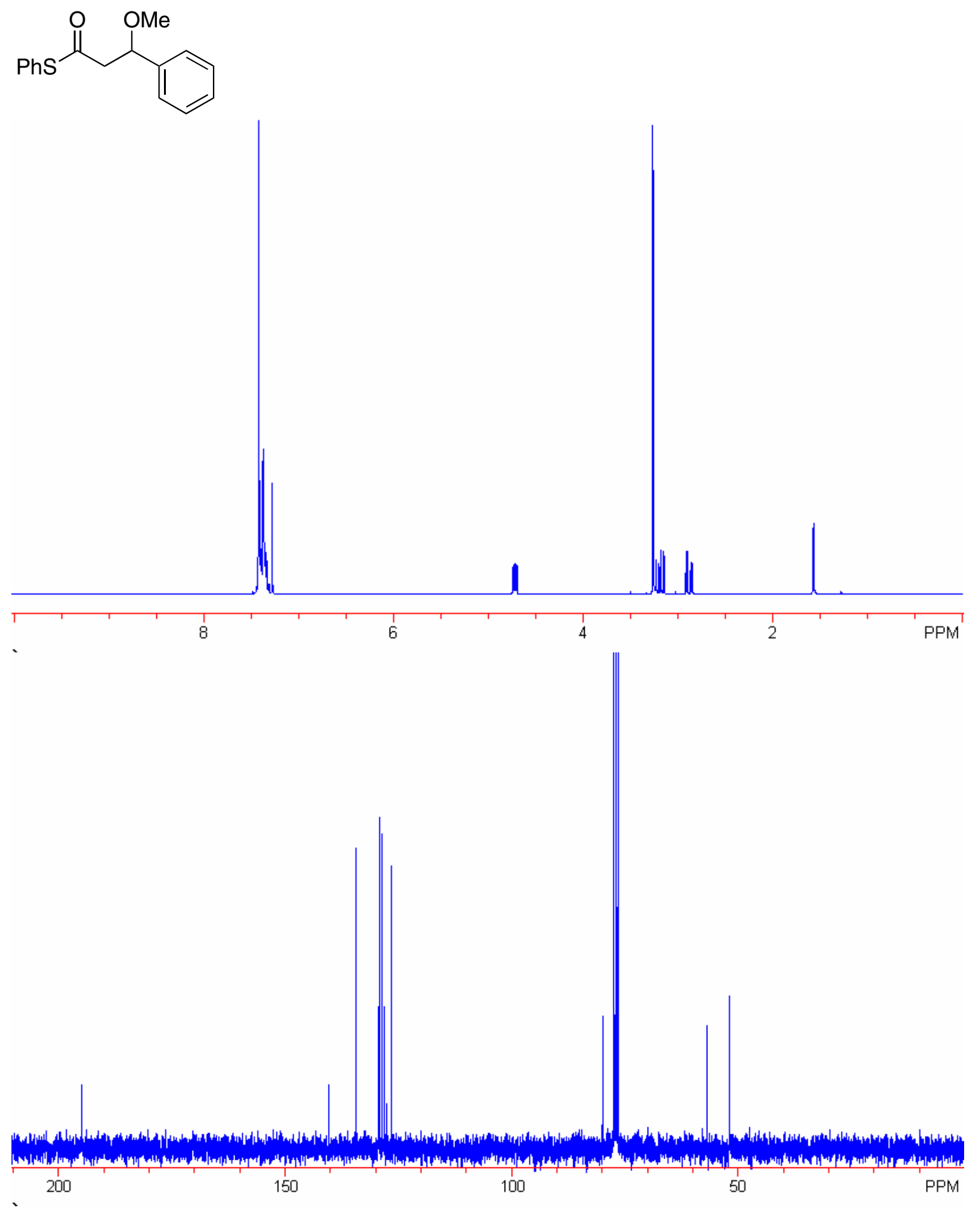

Product 19 

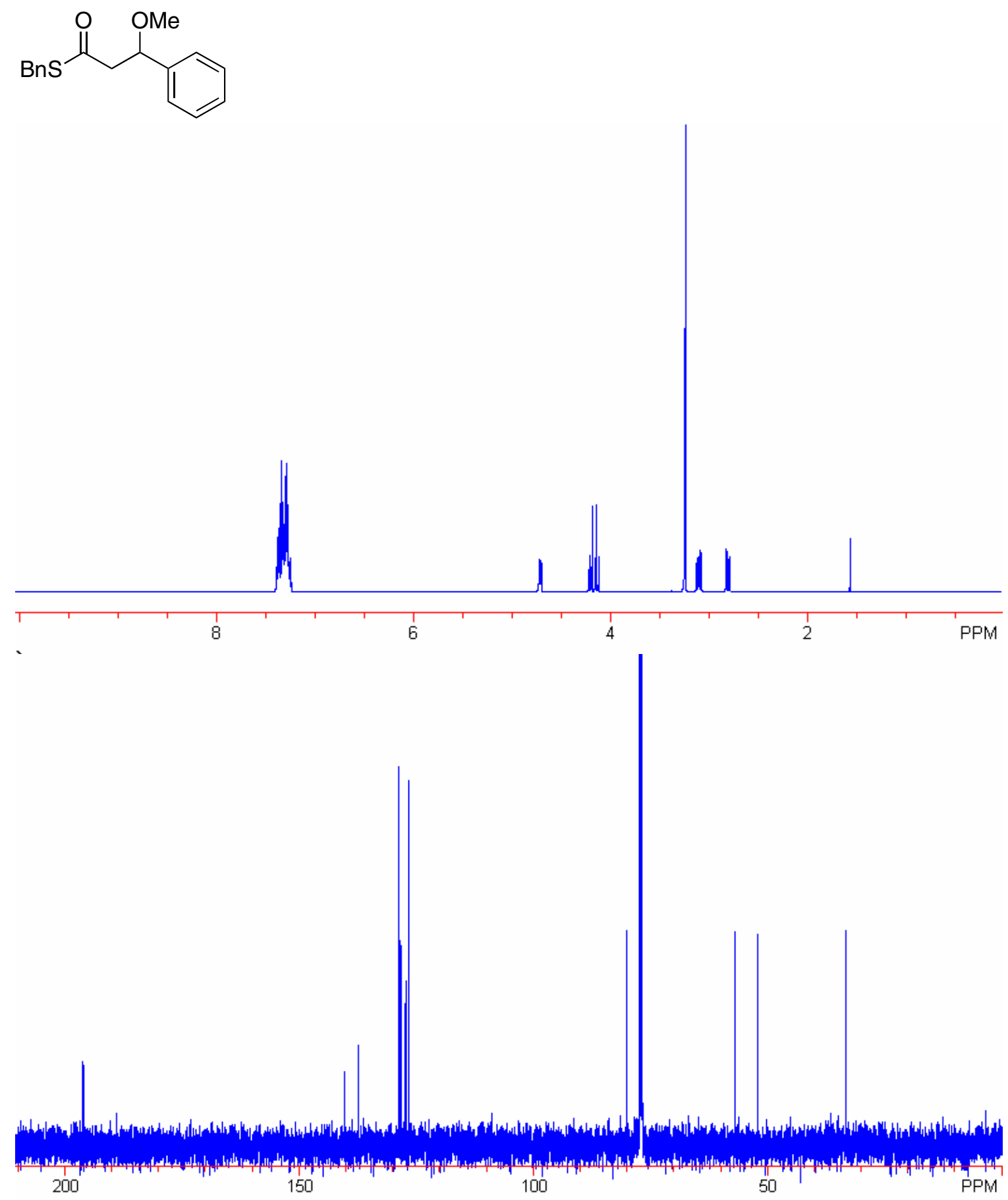

Product 20 
<smiles>COC(C)CC(=O)c1ccc2ccccc2c1</smiles>
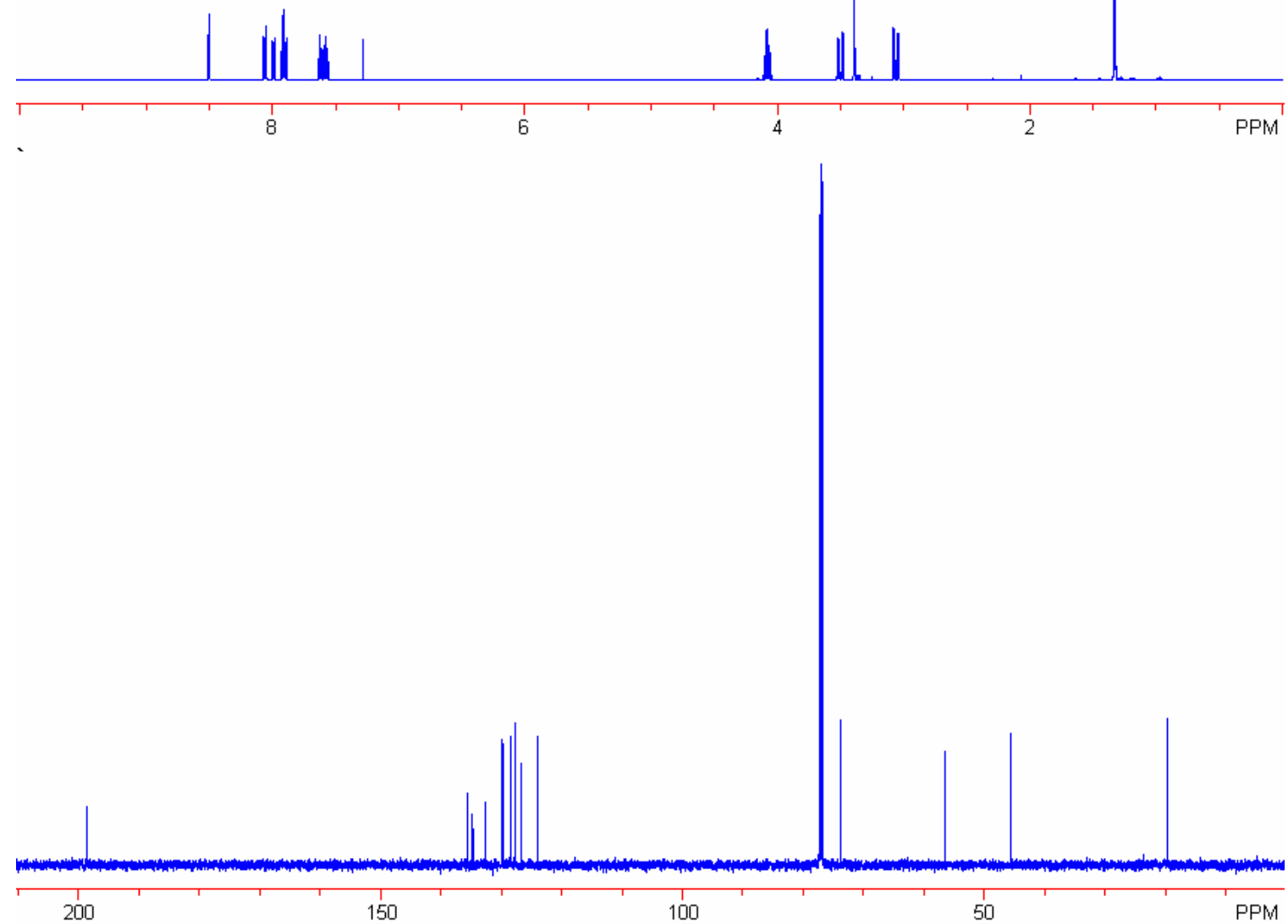

Product 21 
<smiles>COC(C)CC(=O)c1cccc2ccccc12</smiles>

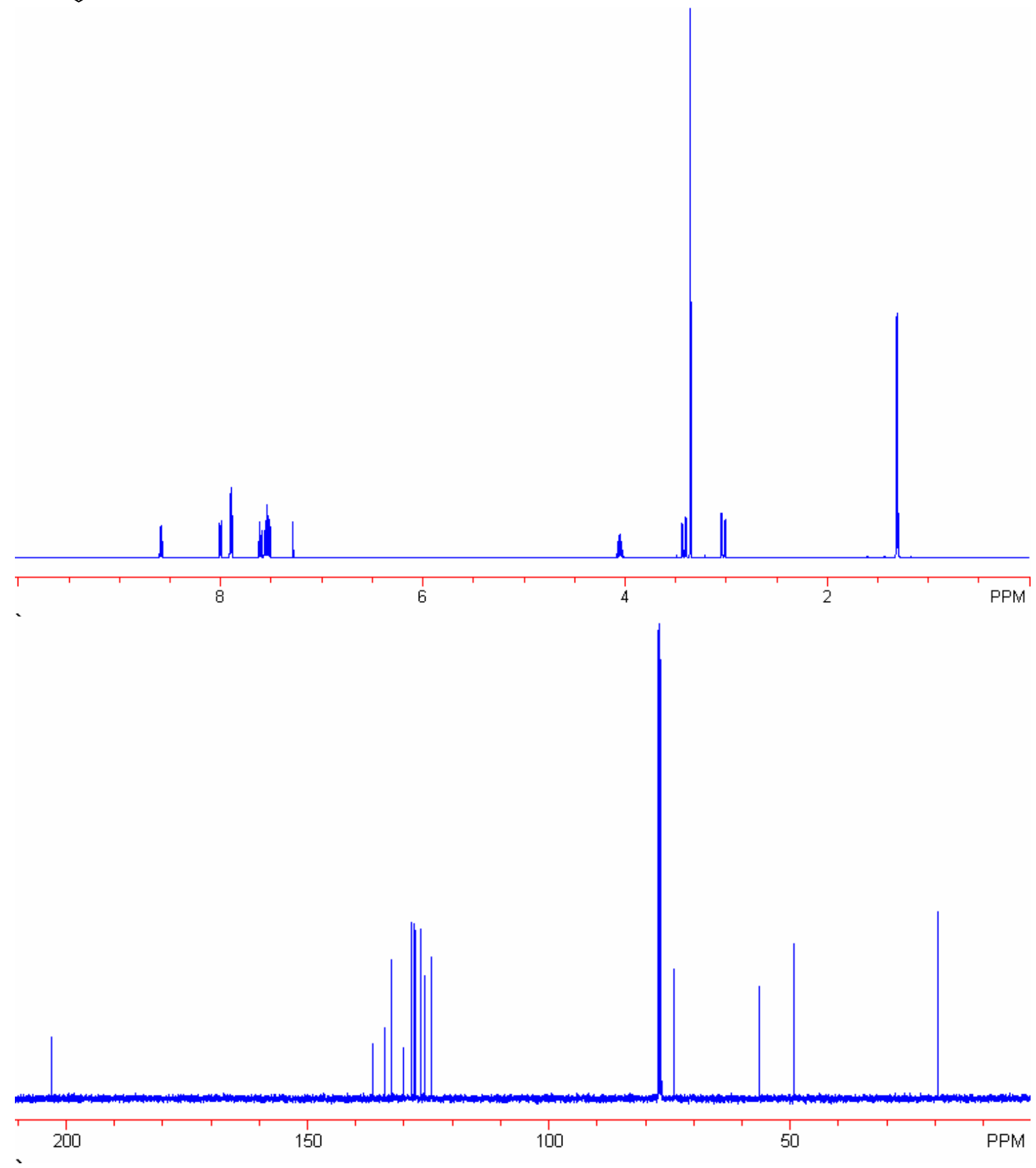

Product 24 
<smiles>COC(C)CC(=O)c1ccccc1</smiles>
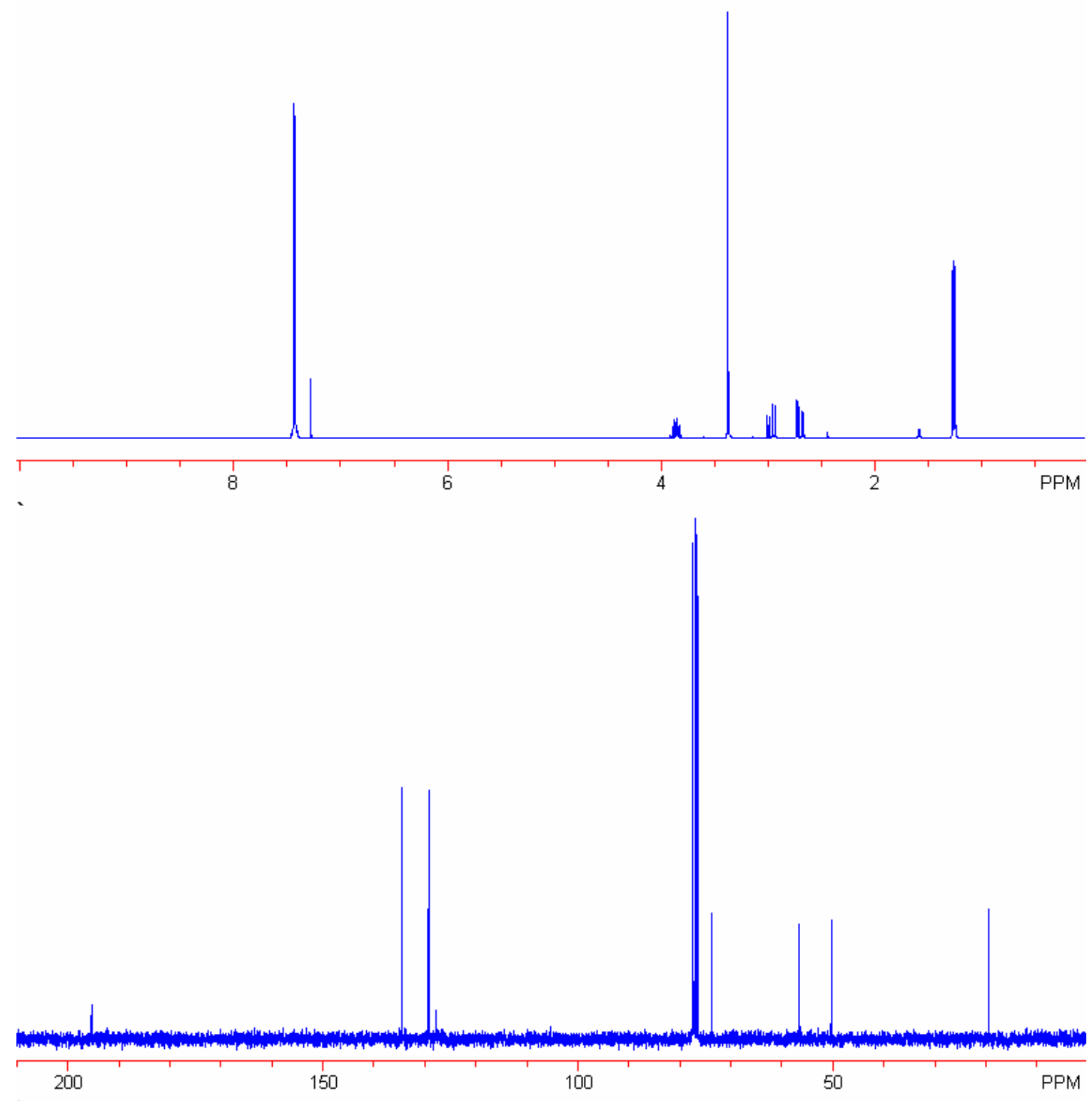

Product 25 

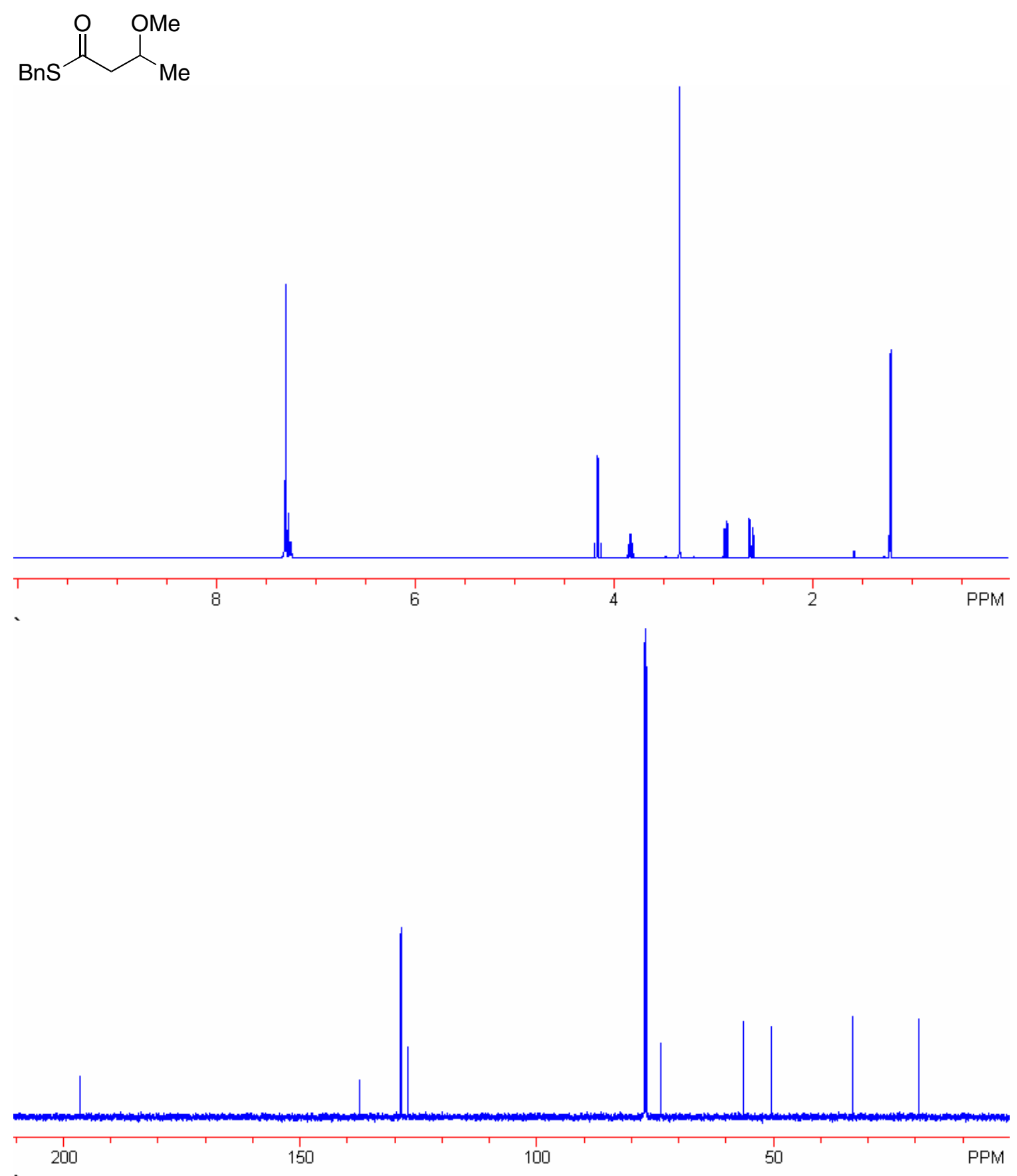

Product 26 


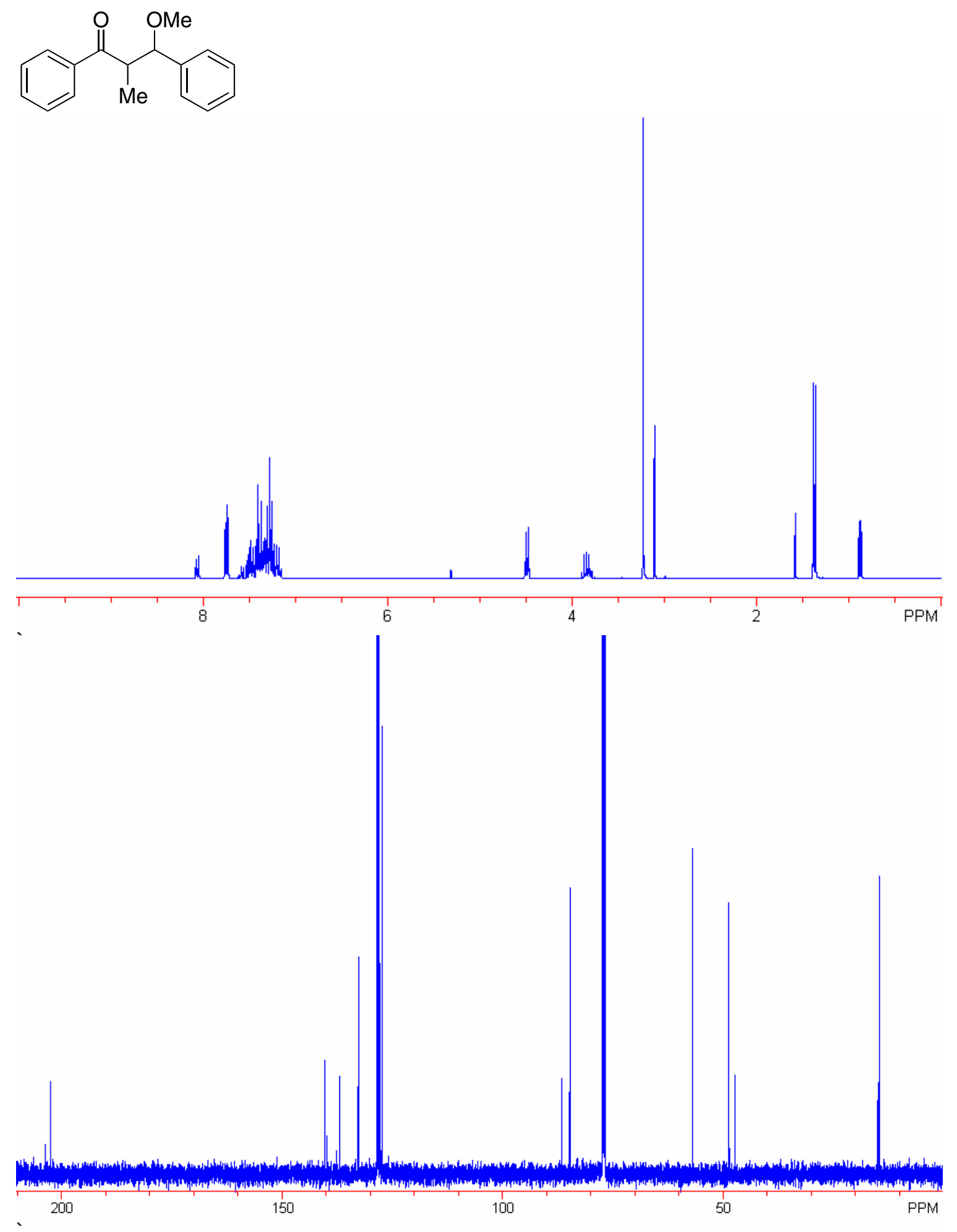

Product 27 
<smiles>COC(C)C(C)C(=O)c1ccccc1</smiles>
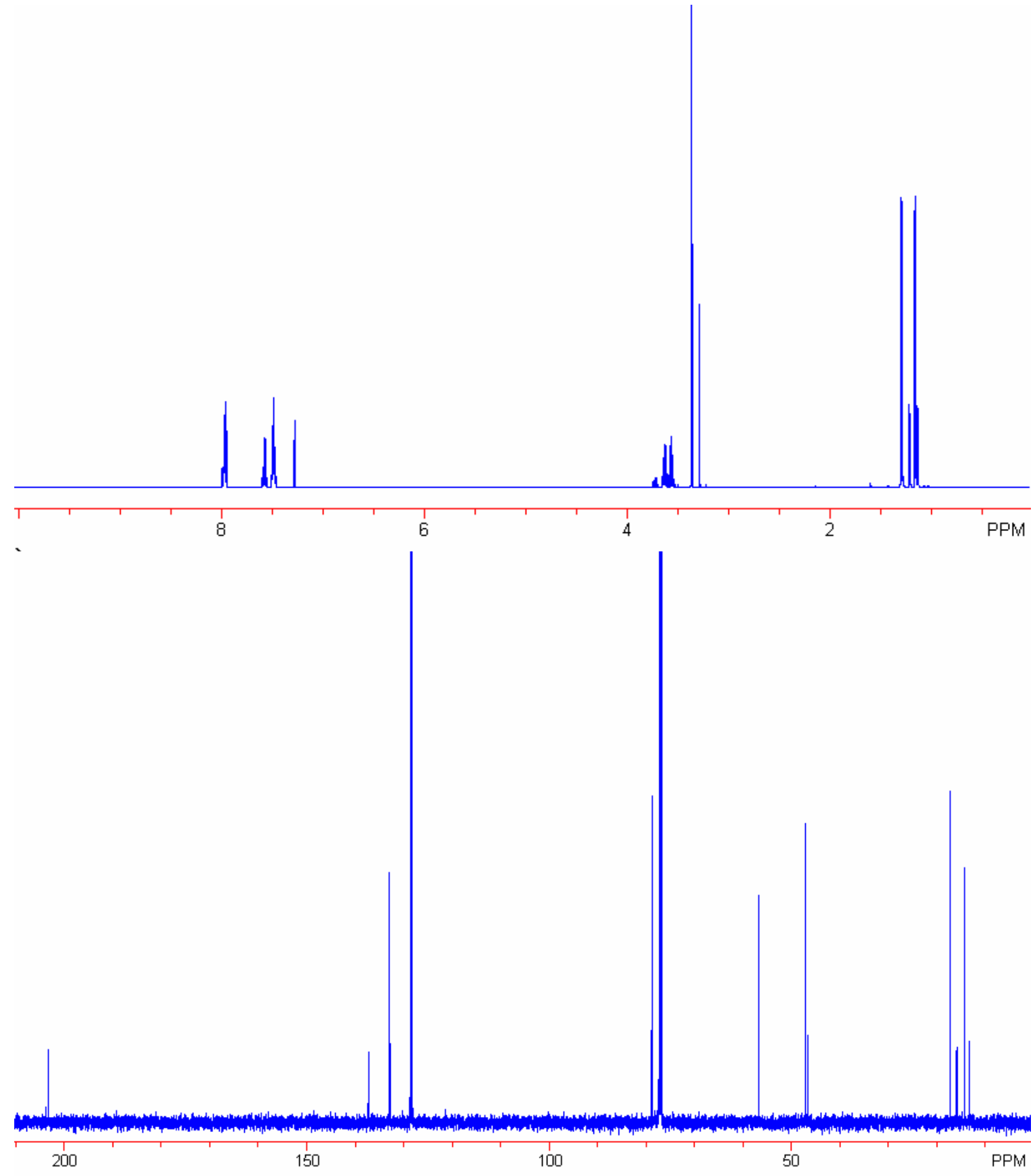